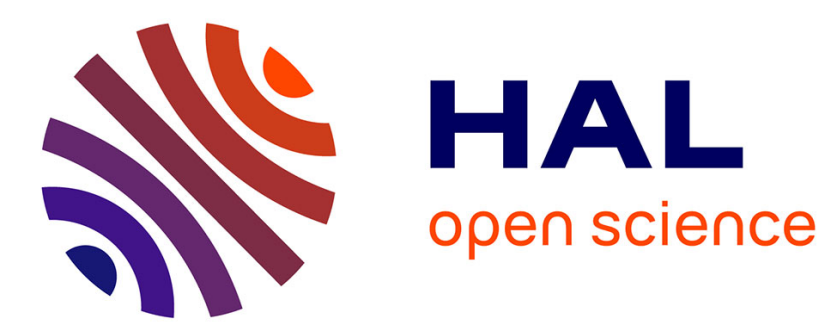

\title{
Regioselective Hydrostannation of Highly Hindered Arylalkynes under Ortho Directing Effects
}

\author{
Abdallah Hamze, Patrick Le Menez, Olivier Provot, Estelle Morvan, \\ Jean-Daniel Brion, Mouad Alami
}

\section{To cite this version:}

Abdallah Hamze, Patrick Le Menez, Olivier Provot, Estelle Morvan, Jean-Daniel Brion, et al.. Regioselective Hydrostannation of Highly Hindered Arylalkynes under Ortho Directing Effects. Tetrahedron, 2010, 66 (45), pp.8698-8706. 10.1016/j.tet.2010.09.010 . hal-02394476

\section{HAL Id: hal-02394476 \\ https://hal.science/hal-02394476}

Submitted on 4 Dec 2019

HAL is a multi-disciplinary open access archive for the deposit and dissemination of scientific research documents, whether they are published or not. The documents may come from teaching and research institutions in France or abroad, or from public or private research centers.
L'archive ouverte pluridisciplinaire HAL, est destinée au dépôt et à la diffusion de documents scientifiques de niveau recherche, publiés ou non, émanant des établissements d'enseignement et de recherche français ou étrangers, des laboratoires publics ou privés. 


\section{Graphical Abstract}

To create your abstract, type over the instructions in the template box below.

Fonts or abstract dimensions should not be changed or altered.

Regioselective Hydrostannation of Highly Hindered
Arylalkynes under Ortho Directing Effects
Abdallah Hamze, Patrick Le Menez, Olivier Provot, Estelle Morvan, Jean-Daniel Brion and Mouâd Alami
Univ Paris-Sud, CNRS, BioCIS, UMR 8076, Laboratoire de Chimie Thérapeutique, Faculté de Pharmacie, rue
J.B. Clément F-92296 Châtenay-Malabry, France.




\title{
Regioselective Hydrostannation of Highly Hindered Arylalkynes under Ortho Directing Effects
}

\author{
Abdallah Hamze, Patrick Le Menez, Olivier Provot, Estelle Morvan, Jean-Daniel Brion and \\ Mouâd Alami* \\ Univ Paris-Sud, CNRS, BioCIS, UMR 8076, Laboratoire de Chimie Thérapeutique, Faculté de Pharmacie, rue J.-B. Clément F-92296 \\ Châtenay-Malabry, France.
}

\begin{abstract}
Palladium-catalyzed hydrostannation reactions of ortho-disubstituted arylalkynes were achieved with total stereo- and regioselectivity in THF at room temperature. The regioselectivity was found to be under the control of the ortho-substituents (ortho-directing effects, ODE) and pure $\alpha$-vinyl stannanes are produced in good yields and as single isomers regardless of the substituents' nature. These hydrostannation $\alpha$-products are precursors of choice for the preparation of stereodefined triarylolefins. (C) 2019 Elsevier Science. All rights reserved
\end{abstract}

Keywords: Alkynes, Palladium, Ortho-Directing Effects, Regioselectivity.

\section{Introduction}

Organostannanes, and particularly vinylstannanes are of increasing importance as synthetic intermediates in organic chemistry due to the large number of carboncarbon bond forming reactions available to these substrates. ${ }^{1}$ As a result of their synthetic utility, considerable effort has been expended toward their preparation $^{2}$ and the addition of tributyltin hydride to alkynes remains the most simple and straightforward route to these vinylstannanes intermediates. ${ }^{3}$ The challenge in the hydrostannation of unsymmetrical alkynes is to directly produce vinylstannanes of high purity with a total regio- and stereoselectivity because mixtures of vinyl stannanes are tedious to separate (destannylation, non polar isomers,...). For palladiumcatalyzed hydrostannation of alkynes, control of stereoselectivity is not a problem since the addition of tributyltin hydride proceeds in a syn manner (cisaddition). ${ }^{2 \mathrm{~d}, 4}$ In contrast, the regiochemical control of this reaction appears to be dependent on steric, ${ }^{2 \mathrm{~d}, 4,5}$ electronic $^{2 \mathrm{~d}}$ and chelating ${ }^{2 \mathrm{~d}, 6}$ factors of the alkyne substituents. We previously described the regioselective palladium-catalyzed hydrostannation of conjugated alkynes. With $(Z)$-enyne $^{7}$ and $(Z)$-enediyne ${ }^{8}$ substrates, the regioselectivity of the $\mathrm{H}-\mathrm{Sn}$ bond addition was found to be totally controlled by the geometry of the double bond (Z-Directing Effect, ZDE) rather than the nature of its substituents $(\mathrm{Cl}$, alkyl, aryl,...) to give exclusively $\alpha$ vinylstannanes (Scheme 1). With ortho substituted internal $^{9}$ and terminal $^{10}$ arylalkyne derivatives, we demonstrated that an ortho substituent delivered the $\mathrm{Bu}_{3} \mathrm{Sn}$ group on the carbon atom $\left(\mathrm{C}_{\alpha}\right)$ whatever the electronic nature of the substituent, electron withdrawing or electron donating (e.g.; $\mathrm{CO}_{2} \mathrm{R}, \mathrm{COR}, \mathrm{OR}, \mathrm{Cl} \ldots$ ). This trend in $\alpha$-regioselectivity was also observed with substrates having ortho nonchelating alkyl substituents (e.g.; $\mathrm{CH}_{3}, i \mathrm{Pr}, \ldots$ ), clearly indicating that coordinating factors were not at the origin of this remarkable regioselectivity (Scheme 1). The results have been rationalized in terms of electronic polarization across the alkyne bond, induced by the ortho substituent whatever its electronic nature. ${ }^{\mathrm{b}}$ Although at the moment, we did not succeed in correlating the $\alpha$-selectivity observed with the triple bond polarization, this ortho-directing effect (ODE) has been successfully extended to control the regiochemistry of the platinum-catalyzed hydrosilylation of internal aliphatic arylalkynes as well as diarylalkynes. ${ }^{11}$

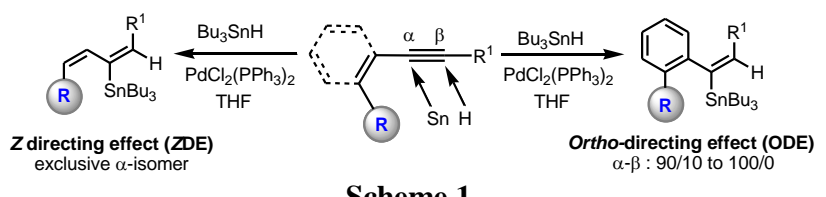

Scheme 1.

Although it was clearly established that the regioselectivity of the $\mathrm{Pd}$-catalyzed hydrostannation of aliphatic alkynes is very sensitive to the steric hindrance, ${ }^{2 \mathrm{~d}, 5 \mathrm{a}, 5 \mathrm{~b}}$ we showed previously in the case of internal ortho substituted diarylalkynes ${ }^{9 b}$ that increasing steric hindrance of ortho substituent provides better $\alpha$ regioselectivities (anti steric effects) as depicted in Scheme 2.
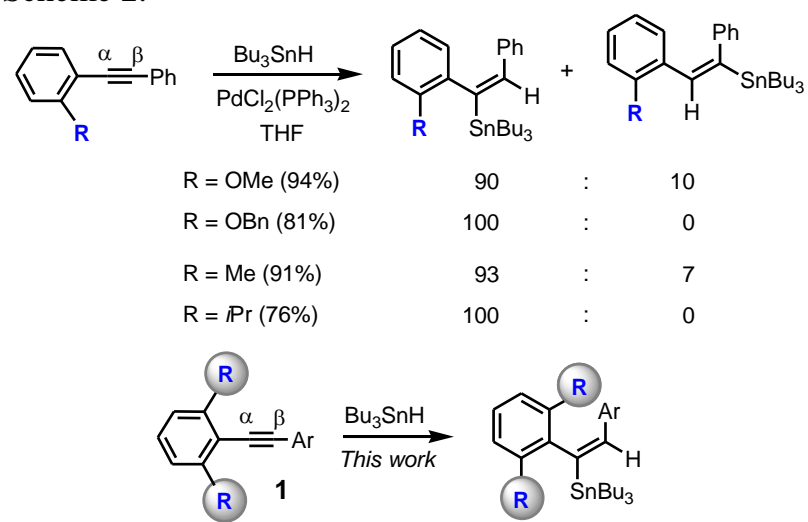

Scheme 2. 
Table 1. Hydrostannation of highly hindered arylalkynes 1 under palladium catalysis.

\begin{tabular}{|c|c|c|c|c|c|c|}
\hline Entry & Alkyne 1 & & $\alpha / \beta$ ratio $^{\mathrm{a}}$ & Vinyl Stannane 2 & & Yield $^{\mathrm{b}}(\%)$ \\
\hline 1 & & $1 \mathrm{a}$ & $90 / 10$ & & $2 \mathbf{a}$ & 94 \\
\hline 2 & & $1 b$ & $100 / 0$ & & $2 \mathbf{b}$ & 86 \\
\hline 3 & & 1c & $100 / 0$ & & $2 c$ & 90 \\
\hline 4 & & 1d & $100 / 0$ & & 2d & 65 \\
\hline 5 & & $1 e$ & $100 / 0$ & & $2 e$ & 71 \\
\hline 6 & & 1f & $100 / 0$ & & $2 f$ & 55 \\
\hline 7 & & $1 \mathrm{~g}$ & $100 / 0$ & & $2 \mathrm{~g}$ & 70 \\
\hline 8 & & $1 \mathrm{~h}$ & $81 / 19$ & & $2 h$ & 86 \\
\hline 9 & & $\mathbf{1 i}$ & $100 / 0$ & & $2 \mathbf{i}$ & 88 \\
\hline 10 & & $\mathbf{1 j}$ & $100 / 0$ & & $2 \mathbf{j}$ & 98 \\
\hline 11 & & $1 k$ & $100 / 0$ & & $2 \mathrm{k}$ & 98 \\
\hline 12 & & 11 & $100 / 0$ & & 21 & 48 \\
\hline 13 & & $1 \mathrm{~m}$ & $95 / 5$ & & $2 m$ & 97 \\
\hline 14 & & 1n & $100 / 0$ & 2 & $2 n$ & 81 \\
\hline 15 & & 10 & $100 / 0$ & & 20 & $91^{\mathrm{c}}$ \\
\hline
\end{tabular}

${ }^{a}$ Ratio was determined by ${ }^{1} \mathrm{H}$ NMR analysis.

${ }^{\mathrm{b}}$ Yields were given for isolated products.

c 3.0 equiv. of $\mathrm{Bu}_{3} \mathrm{SnH}$ were used. 
These findings clearly highlighted the role of steric hindrance of substituent in directing the $\alpha$-addition of the metals to the more hindered alkyne carbon atom $(\mathrm{C} \alpha)$. To the best of our knowledge there has been no systematic study on metal-catalyzed hydrometallation of highly hindered arylalkynes $\mathbf{1}$ having ortho/ortho' substituents. Herein, the results of these studies are reported using tributyltin hydride or triethylsilane in order to determine the impact of ortho/ortho' substituents of $\mathbf{1}$ on the $\alpha$ regioselectivity.

\section{Results and Discussion}

At the beginning of this work, hindered ortho/ortho'diarylalkynes 1 were prepared according to SonogashiraLinstrumelle (SL) couplings ${ }^{12}$ using $\mathrm{PdCl}_{2}\left(\mathrm{PPh}_{3}\right)_{2}$ $\mathrm{mol} \%), \mathrm{CuI}(10 \mathrm{~mol} \%)$, piperidine as the base in THF, in good to excellent yields. The hydrostannation of diarylalkynes 1 was conducted under standard conditions using $\mathrm{PdCl}_{2}\left(\mathrm{PPh}_{3}\right)_{2}(5 \mathrm{~mol} \%)$ and $\mathrm{Bu}_{3} \mathrm{SnH}$ (1.3 equiv) in THF. The results of this study are summarized in Table 1. In order to establish a baseline regioselectivity control, 1(2-methoxyphenyl)-2-phenylacetylene 1a bearing a single ortho $\mathrm{MeO}$ substituent was evaluated. Under the above conditions, 1a was hydrostannylated to give a diastereomeric mixture of non separable $\alpha$ and $\beta$ vinylstannanes with an excellent but not total $\alpha$-preference (entry $1, \alpha-\mathbf{1 a} / \beta-2 \mathbf{a}: 90 / 10)$. For comparison, by switching the $\mathrm{MeO}$ substituent from the ortho to the para position on the aromatic, an equimolar isomeric $\alpha-2 / \beta-2$ product distribution was obtained (data not shown). More interestingly, hydrostannation of ortho-disubstituted arylalkyne 1b, 1c and 1d bearing on the same aromatic nucleus two ortho electron-donating substituents was totally regioselective ${ }^{13}$ furnishing $\alpha$-vinylstannanes $\mathbf{2 b}$-d in good yields (entries 2-4). As expected for electronic considerations, hydrostannation of ortho/ortho' disubstituted diarylalkynes 1e-g, bearing a para $\mathrm{MeO}$ substituent on the second aromatic nucleus, furnished vinylstannanes $\mathbf{2 e - g}$, again with a total $\alpha$-regioselectivity (entries 5-7). The example depicted in entry 8 with "pushpull" diarylalkyne $\mathbf{1 h}$ is particularly interesting. In this case, where ODE is opposed by a strong electronic effect (induced by the para-CN), the $\alpha$-regioselectivity was less spectacular and $\beta$-vinylstannane was obtained as minor products together with $\alpha$-adduct (entry $8, \alpha-2 \mathbf{h} / \beta-2 \mathbf{h}$ : $81 / 19)$. By replacing the ortho and ortho' electron-donating substituents with chlorine atoms, the $\alpha$-regioselectivity is entirely governed by ODE rather than the $p$-CN induced polarization of the triple bond (entry 9). For substrate $\mathbf{1 j}-\mathbf{1 1}$, ODE and electronic factors cooperate to give $\alpha$-substituted vinylstannanes $\mathbf{2} \mathbf{j}-\mathbf{l}$ exclusively in good yields (entries 10 12). We next tested the ortho-directing effect on the distribution products with arylalkynol $\mathbf{1 m}$. It was found that hydrostannation of $\mathbf{1} \mathbf{m}$ proceeded with an excellent $\alpha$ regioselectivity in a nearly quantitative yield (entry 13, $\alpha$ $\mathbf{2 m} / \boldsymbol{\beta}-\mathbf{2 m}$ : 95/5). As it was observed for diarylalkynes $\mathbf{1 b}-\mathbf{g}$, 1i-l, the hydrostannation of arylalkynols $\mathbf{1 m}$ and $\mathbf{1 n}$ bearing two substituents on the ortho position was totally regioselective and produced $\alpha$-vinyl stannanes $\mathbf{2 m}$ and $\mathbf{2 n}$ in good yields. The last example, described in entry 15 sums up this study. The two triple bonds of alkyne 10 were successfully hydrostannylated with a total regioselectivity

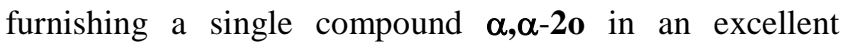
isolated yield. In this case, if ortho substituents are at the origin of the $\alpha$-regioselectivity observed for the hydrostannation of the hindered alkyne, the exclusive $\alpha$ stannylation of the alkynol moiety is not immediately clear.

The success of this ortho/ortho'-substituent regiocontrol prompted additional investigations with ortho-disubstituted terminal aryl alkynes. The results of this study are abstracted in Scheme 3. Hydrostannation of electron-rich arylalkyne 1p as well as electron deficient arylalkyne 1q was achieved and again, a total regioselectivity was observed. In these cases, exclusive $\alpha$-isomers $\mathbf{2 p}$ and $\mathbf{2 q}$ were produced in excellent yields (2p: 92\%, 2q: 98\%).

Scheme 3 Hydrostannation of terminal ortho/ortho' disubstituted arylalkynes $\mathbf{1 p}$ and $\mathbf{1 q}$.
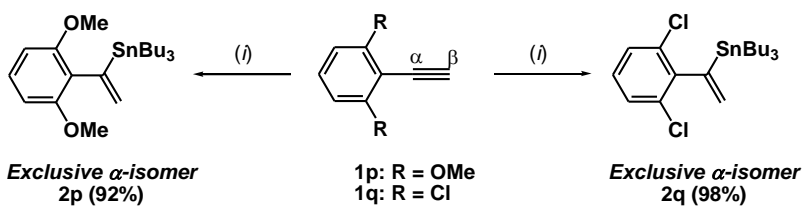

Reagents and conditions. (i) $\mathrm{Bu} 3 \mathrm{SnH}$ (1.3 equiv), $\mathrm{PdCl}_{2}\left(\mathrm{PPh}_{3}\right)_{2}$ $(5 \mathrm{~mol} \%), 20^{\circ} \mathrm{C}$, THF, $0.5 \mathrm{~h}$.

Next, to examine the synthetic utility of $\alpha$-vinylstannanes of type 2, we first evaluated the Stille coupling of 4iodoanisole with $\mathbf{2 k}$ and 2o. However, the coupling products $\mathbf{4 a}$ and $\mathbf{4 b}$ were not obtained in satisfactory yields. Therefore, a second synthetic approach involving an iododestannylation-Negishi sequence was explored (Scheme 4). ${ }^{14}$ Thus, stereospecific iodo-destannylation of 2k and 20 with molecular iodine (1 equiv) in $\mathrm{CH}_{2} \mathrm{Cl}_{2}$ at $20^{\circ} \mathrm{C}$ provided cleanly and rapidly the suitable electrophilic vinyl iodides $\mathbf{3 a}$ and $\mathbf{3} \mathbf{b}$ in quantitative yields. Further coupling with 4-methoxyarylzinc chloride in the presence of $\mathrm{PdCl}_{2}\left(\mathrm{PPh}_{3}\right)_{2} \quad(5 \mathrm{~mol} \%)$ furnished the triarylated ethylenic compounds $\mathbf{4 a - b}$ with a total stereospecificity in good yields (Scheme 4).

Finally, we wanted to examine if this total $\alpha$-selectivity could be successfully extended to the hydrosilylation of various ortho-disubstituted alkynes. Preliminary results are presented in Table 2. Reaction of ortho-disubstituted diarylalkyne $\mathbf{1 b}$ with triethylsilane in THF in the presence of $\mathrm{PtO}_{2}(5 \mathrm{~mol} \%)$ at $70{ }^{\circ} \mathrm{C}$ for $1 \mathrm{~h}$ afforded regioselectively an inseparable $90 / 10$ mixture of the vinylsilanes $\boldsymbol{\alpha}-\mathbf{3 a}$ and $\boldsymbol{\alpha}-\mathbf{3 b}$ in $90 \%$ yield (entry 1 , Table 2 ). For comparison, the hydrosilylation of alkyne $\mathbf{1 a}$ bearing a single $\mathrm{MeO}$ substituent on the ortho position, (baseline control) 
afforded a mixture of $\alpha$ - and $\beta$-vinylsilanes but with a lower $\alpha$-selectivity $(\alpha / \beta$ : $80 / 20)$.

Scheme 4 Iododestannylation of $\mathbf{2 k}$ and $\mathbf{2 0}$ followed by Negishi Cross-coupling reactions under palladium catalysis.
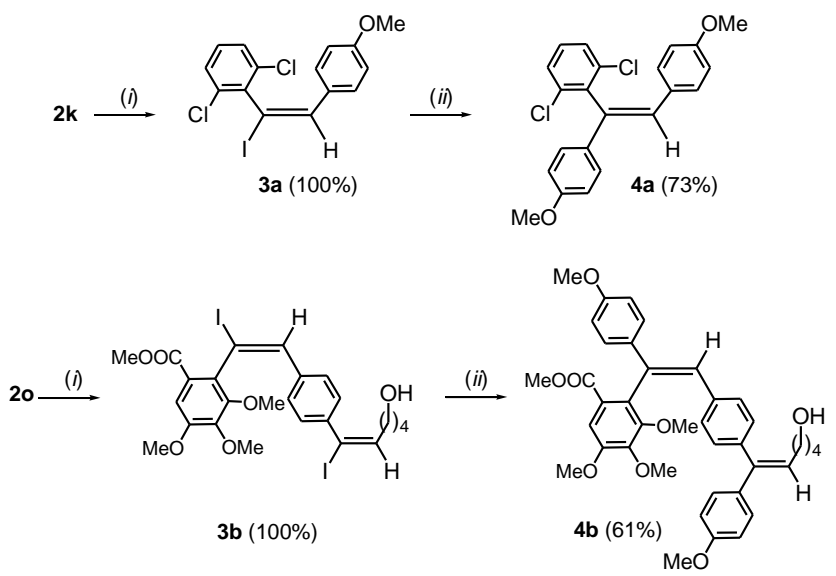

Reagents and conditions. (i) $\mathrm{I}_{2}, \mathrm{CH}_{2} \mathrm{Cl}_{2}$. (ii) $4-\mathrm{MeOC}_{6} \mathrm{H}_{4} \mathrm{ZnCl}$, $\mathrm{PdCl}_{2}\left(\mathrm{PPh}_{3}\right)_{2} 5 \mathrm{~mol} \%$, THF.

A similar $\alpha$-preference was also observed with alkyne 1e bearing two MeO-substituents on the ortho and ortho' position (97\%, entry 2). As previously observed for the hydrostannation of $\mathbf{1 j}$ (entry 10, Table 1), the hydrosilylation of $\mathbf{1} \mathbf{j}$ bearing two chlorine atoms on the ortho positions was totally regioselective. Again, electronic factors, generated by the chlorine atoms, and ODE cooperate and the reaction produced a single $\alpha$-vinylsilane $3 \mathbf{c}$ in a nearly quantitative yield ( $98 \%$, entry 3 , Table 2$)$.

Table 2 Hydrosilylation of ortho disubstituted arylalkynes $\mathbf{1}$.
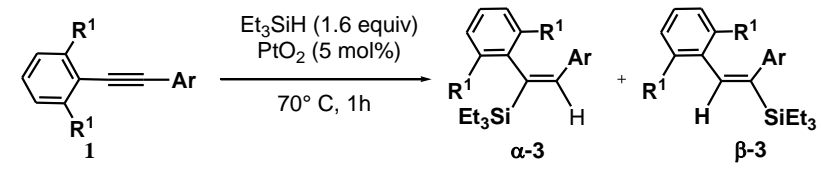

\begin{tabular}{|c|c|c|c|c|}
\hline Entry & $\begin{array}{c}\text { Alkyne } \\
1\end{array}$ & $\begin{array}{c}\text { Selectivity } \\
\alpha / \beta^{\mathrm{a}}\end{array}$ & $\begin{array}{c}\text { Vinylsilane } \\
\alpha-3\end{array}$ & $\begin{array}{c}\text { Yield }^{b} \\
(\%)\end{array}$ \\
\hline 1 & $1 b$ & $90 / 10$ & & 90 \\
\hline 2 & 1e & $92 / 8$ & & 97 \\
\hline 3 & $\mathbf{1 j}$ & $100 / 0$ & & 98 \\
\hline
\end{tabular}

\footnotetext{
${ }^{a}$ Ratio was determined by ${ }^{1} \mathrm{H}$ NMR analysis.

${ }^{\mathrm{b}}$ Yields were given for isolated products.
}

\section{Conclusion}

In summary, we demonstrated that hydrostannation of hindered arylalkynes bearing on the same aromatic nucleus two ortho-substituents give exclusively the $\alpha$ vinylstannanes in good yields whatever the electronic nature and steric hindrance of substituents. Moreover, preliminary results indicate that the $\mathrm{PtO}_{2}$-catalyzed hydrosilylation of these hindered alkynes was also highly regioselective. The factors governing this total $\alpha$-selectivity would be close to those observed in the hydrostannation or hydrosilylation of arylalkynes bearing on the ortho position a single substituent (ODE). This study shows that it is possible to predict the exclusive $\alpha$-isomers formation even if the exact origin of this regioselectivity remains unclear. Interestingly, the products of the hydrostannation reaction can be efficiently used as versatile substrates in iododestannylation-Negishi sequences to afford stereodefined triarylolefins of therapeutic interest. ${ }^{15}$

\section{Experimental}

\section{General comments}

All glassware were oven-dried at $140^{\circ} \mathrm{C}$ and all reactions were conducted under a nitrogen atmosphere. Solvents: cyclohexane, ethyl acetate (EtOAc), for chromatography were technical grade. Diethylether $\left(\mathrm{Et}_{2} \mathrm{O}\right)$ and tetrahydrofuran (THF) were distilled under argon from sodium-benzophenone ketyl, piperidine, triethylamine from potassium hydroxide. $\mathrm{PdCl}_{2}\left(\mathrm{PPh}_{3}\right)_{2}$ and $\mathrm{Bu}_{3} \mathrm{SnH}^{16}$ were prepared following litterature procedure. All new compounds were characterized by ${ }^{1} \mathrm{H}-\mathrm{NMR},{ }^{13} \mathrm{C}-\mathrm{NMR}$, IR spectroscopy and elemental analysis. ${ }^{1} \mathrm{H}$ and ${ }^{13} \mathrm{C}$ NMR spectra were measured in $\mathrm{CDCl}_{3}$ with a Bruker ARX 400 or Bruker Avance 300. ${ }^{1} \mathrm{H}$ chemical shifts are reported in ppm from an internal standard TMS or of residual chloroform (7.27 ppm). The following abreviation are used: $\mathrm{m}$ (multiplet), s (singlet), br s (broad singlet), d (doublet), $\mathrm{t}$ (triplet) dd (doublet of doublet), q (quadruplet), sextet (sextuplet). ${ }^{13} \mathrm{C}$ chemical shifts are reported in ppm from the central peak of deuteriochloroform (77.14). IR spectra were measured on a Bruker Vector 22 spectrophotometer (neat, $\mathrm{cm}^{-1}$ ). Elemental analyses were performed with a Perkin-Elmer 240 analyzer. Analytical TLC was performed on Merck precoated silica gel 60 F-254 plates. Merck silica gel 60 (230-400 mesh) was used for column chromatography. The plates were visualized by either UV light $(254 \mathrm{~nm})$, or by a solution of phosphomolybdic acid in ethanol. Melting points (m.p.) were recorded on a Büchi B450 apparatus and were uncorrected.

\section{General procedure for the synthesis of diarylalkynes 1}

\section{procedure for the synthesis of diarylalkynes (A)}

To a flame-dried flask under a nitrogen atmosphere were added $\mathrm{PdCl}_{2}\left(\mathrm{PPh}_{3}\right)_{2}(120 \mathrm{mg}, 0.17 \mathrm{mmol}), \mathrm{CuI}$ (76 mg, 0.4 
mmol), aryliodide (4.0 mmol), piperidine (1.2 $\mathrm{mL}, 12.0$ $\mathrm{mmol})$ in THF $(12 \mathrm{~mL})$. To this solution was added dropwise 1-alkyne $(6.0 \mathrm{mmol})$. After stirring at room temperature for $12 \mathrm{~h}$, the mixture was diluted in $\mathrm{Et}_{2} \mathrm{O}(25$ $\mathrm{mL}$ ), and then washed with a saturated $\mathrm{NH}_{4} \mathrm{Cl}$ solution (20 $\mathrm{mL})$. After extraction with $\mathrm{Et}_{2} \mathrm{O}(25 \mathrm{ml})$ the combined organic layers were dried over $\mathrm{MgSO}_{4}$. After concentration under reduced pressure, the residue was purified by column chromatography over silica gel, to yield the desired orthodisubstituted alkyne $\mathbf{1}$.

\section{1,3-Bis(benzyloxy)-2-(phenylethynyl)benzene (1c)}

White solid, mp: $106-108{ }^{\circ} \mathrm{C} . \quad(90 \%) . \quad \mathrm{R}_{\mathrm{f}}=0.23$ $\left(\mathrm{Et}_{2} \mathrm{O} / \mathrm{Cyclohexane}, 5 / 95, \mathrm{SiO}_{2}\right) .{ }^{1} \mathrm{H} \mathrm{NMR}\left(\mathrm{CDCl}_{3}, 300\right.$ $\mathrm{MHz}): \delta 5.14(4 \mathrm{H}, \mathrm{s}), 6.54(2 \mathrm{H}, \mathrm{d}, J=8.4 \mathrm{~Hz}), 7.11(1 \mathrm{H}, \mathrm{t}$, $J=8.4 \mathrm{~Hz}), 7.15-7.54(15 \mathrm{H}, \mathrm{m}) .{ }^{13} \mathrm{C} \mathrm{NMR}(75 \mathrm{MHz}$, $\left.\mathrm{CDCl}_{3}\right): \delta_{\mathrm{C}} 70.6\left(2 \mathrm{CH}_{2}\right), 82.6(\mathrm{C}), 98.4(\mathrm{C}), 103.7(\mathrm{C})$, $106.0(2 \mathrm{CH}), 124.3(\mathrm{C}), 126.9(4 \mathrm{CH}), 127.8(2 \mathrm{CH}), 127.9$ $(\mathrm{CH}), 128.3(2 \mathrm{CH}), 128.5(4 \mathrm{CH}), 129.6(\mathrm{CH}), 131.6$ (2CH), 137.2 (2C), 160.6 (2C). IR ( $\mathrm{cm}^{-1}$, neat): 1582, 1474, 1446, 1375, 1261, 1116, 771, 732, 690. Anal. Calcd for $\mathrm{C}_{28} \mathrm{H}_{22} \mathrm{O}_{2}$ (390.47): $\mathrm{C} 86.13, \mathrm{H} 5.68$, found $\mathrm{C} 85.91, \mathrm{H}$ 5.70 .

\section{2-(Phenylethynyl)-1,3-phenylene diacetate (1d)}

Brown solid, mp: 106-108 ${ }^{\circ} \mathrm{C} . \quad(65 \%) . \quad \mathrm{R}_{\mathrm{f}}=0.40$ $\left(\mathrm{Et}_{2} \mathrm{O} / \mathrm{Cyclohexane}, 30 / 70, \mathrm{SiO}_{2}\right) .{ }^{1} \mathrm{H} \mathrm{NMR}\left(\mathrm{CDCl}_{3}, 300\right.$ $\mathrm{MHz}): \delta 2.37(6 \mathrm{H}, \mathrm{s}), 7.06(2 \mathrm{H}, \mathrm{d}, J=8.2 \mathrm{~Hz}), 7.40-7.32$ (4H, m), 7.51-7.43 (2H, m). $\left.{ }^{13} \mathrm{C} \mathrm{NMR} \mathrm{(75} \mathrm{MHz,} \mathrm{CDCl}_{3}\right): \delta$ $20.9\left(2 \mathrm{CH}_{3}\right), 79.7(\mathrm{C}), 98.8(\mathrm{C}), 112.5(\mathrm{C}), 119.9(2 \mathrm{CH})$, $122.7(\mathrm{C}), 128.5(2 \mathrm{CH}), 128.9(\mathrm{CH}), 129.2(\mathrm{CH}), 131.5$ (2CH), 152.3 (2C), 168.5 (2C). IR ( $\mathrm{cm}^{-1}$, neat): 1761, 1494, 1457, 1371, 1182, 1026, 882, 758, 690. Anal. Calcd for $\mathrm{C}_{18} \mathrm{H}_{14} \mathrm{O}_{4}$ (294.30): C 73.46, $\mathrm{H}$ 4.79, found $\mathrm{C} 73.74, \mathrm{H}$ 4.65 .

1,3-Dimethoxy-2-((4-methoxyphenyl)ethynyl)benzene (1e) Brown solid, mp:142-144 ${ }^{\circ} \mathrm{C} . \quad(71 \%) ; \quad \mathrm{R}_{\mathrm{f}}=0.15$ $\left(\mathrm{Et}_{2} \mathrm{O} / \mathrm{Cyclohexane}, 10 / 90, \mathrm{SiO}_{2}\right) .{ }^{1} \mathrm{H} \mathrm{NMR}\left(\mathrm{CDCl}_{3}, 300\right.$ $\mathrm{MHz}): \delta 3.82(3 \mathrm{H}, \mathrm{s}), 3.91(6 \mathrm{H}, \mathrm{s}), 6.56(2 \mathrm{H}, \mathrm{d}, J=8.4 \mathrm{~Hz})$, $6.86(2 \mathrm{H}, \mathrm{d}, J=8.4 \mathrm{~Hz}), 7.24(1 \mathrm{H}, \mathrm{t}, J=8.4 \mathrm{~Hz}), 7.53(2 \mathrm{H}$, $\mathrm{d}, J=8.4 \mathrm{~Hz}) \cdot{ }^{13} \mathrm{C} \mathrm{NMR}\left(75 \mathrm{MHz}, \mathrm{CDCl}_{3}\right): \delta 55.3\left(\mathrm{CH}_{3}\right)$, $56.2\left(2 \mathrm{CH}_{3}\right), 80.4(\mathrm{C}), 97.9(\mathrm{C}), 102.0(\mathrm{C}), 103.6(2 \mathrm{CH})$, $113.9(2 \mathrm{CH}), 116.2(\mathrm{C}), 129.5(\mathrm{CH}), 133.2(2 \mathrm{CH}), 159.4$ (C), 161.3 (2C). IR ( $\mathrm{cm}^{-1}$, neat): 1579, 1513, 1472, 1252, 1103, 826, 778, 726. Anal. Calcd for $\mathrm{C}_{17} \mathrm{H}_{16} \mathrm{O}_{3}$ (268.31): C 76.10, H 6.01, found C 76.25, H 5.84.

(2-((4-Methoxyphenyl)ethynyl)-1,3-phenylene) bis(oxy)bis (methylene)dibenzene (1f)

Brown solid, mp: 89-91 ${ }^{\circ} \mathrm{C} . \quad(55 \%) ; \quad \mathrm{R}_{\mathrm{f}}=0.20$ $\left(\mathrm{Et}_{2} \mathrm{O} / \mathrm{Cyclohexane}, 5 / 95, \mathrm{SiO}_{2}\right) .{ }^{1} \mathrm{H} \mathrm{NMR}\left(\mathrm{CDCl}_{3}, 300\right.$ $\mathrm{MHz}): \delta 3.84(3 \mathrm{H}, \mathrm{s}), 5.21(4 \mathrm{H}, \mathrm{s}), 6.61(2 \mathrm{H}, \mathrm{d}, J=8.3 \mathrm{~Hz})$, $6.87(2 \mathrm{H}, \mathrm{d}, J=8.8 \mathrm{~Hz}), 7.17(1 \mathrm{H}, \mathrm{t}, J=8.3 \mathrm{~Hz}), 7.66-7.25$ $(12 \mathrm{H}, \mathrm{m}) .{ }^{13} \mathrm{C}$ NMR $\left(75 \mathrm{MHz}, \mathrm{CDCl}_{3}\right): \delta 55.4\left(\mathrm{CH}_{3}\right), 70.7$ $\left(2 \mathrm{CH}_{2}\right), 81.1(\mathrm{C}), 98.4(\mathrm{C}), 104.1(\mathrm{C}), 106.1(2 \mathrm{CH}), 114.0$ $(2 \mathrm{CH}), 116.6(\mathrm{C}), 127.0(4 \mathrm{CH}), 127.8(2 \mathrm{CH}), 128.5(4 \mathrm{CH})$,
$129.2(\mathrm{CH}), 133.0(2 \mathrm{CH}), 137.3(2 \mathrm{C}), 159.4(\mathrm{C}), 160.4$ (2C). IR ( $\mathrm{cm}^{-1}$, neat): 1574, 1509, 1449, 1378, 1247, 1087, 1028, 830, 780, 727, 692. Anal. Calcd for $\mathrm{C}_{29} \mathrm{H}_{24} \mathrm{O}_{3}$ (420.50): C 82.83, H 5.75, found C 82.72, H 5.64.

2-((4-Methoxyphenyl)ethynyl)-1,3-phenylene diacetate (1g) (in this case TEA was used as base instead piperidine). Brown solid, mp: 117- $119{ }^{\circ} \mathrm{C} . \quad(85 \%) . \quad \mathrm{R}_{\mathrm{f}}=0.45$ $\left(\mathrm{Et}_{2} \mathrm{O} / \mathrm{Cyclohexane}, 30 / 70, \mathrm{SiO}_{2}\right) .{ }^{1} \mathrm{H}$ NMR $\left(\mathrm{CDCl}_{3}, 300\right.$ $\mathrm{MHz}): \delta 2.36(6 \mathrm{H}, \mathrm{s}), 3.83(3 \mathrm{H}, \mathrm{s}), 6.88(2 \mathrm{H}, \mathrm{d}, J=8.8 \mathrm{~Hz})$, $7.04(2 \mathrm{H}, \mathrm{d}, J=8.0 \mathrm{~Hz}), 7.35(1 \mathrm{H}, \mathrm{t}, J=8.0 \mathrm{~Hz}), 7.40(2 \mathrm{H}$, $\mathrm{d}, J=8.8 \mathrm{~Hz}) \cdot{ }^{13} \mathrm{C} \mathrm{NMR}\left(75 \mathrm{MHz}, \mathrm{CDCl}_{3}\right): \delta 20.9\left(2 \mathrm{CH}_{3}\right)$, $55.4\left(\mathrm{CH}_{3}\right), 78.4(\mathrm{C}), 99.0(\mathrm{C}), 112.8(\mathrm{C}), 114.2(2 \mathrm{CH})$, $114.8(\mathrm{C}), 119.9(2 \mathrm{CH}), 128.8(\mathrm{CH}), 133.1(2 \mathrm{CH}), 152.2$ (2C), 160.2 (C), 168.6 (2C). IR ( $\mathrm{cm}^{-1}$, neat): 1756, 1515, 1458, 1374, 1190, 1029, 882, 836, 734. Anal. Calcd for $\mathrm{C}_{19} \mathrm{H}_{16} \mathrm{O}_{5}$ (324.33): C 70.36, $\mathrm{H} 4.97$, found $\mathrm{C} 70.01, \mathrm{H}$ 4.75 .

\section{4-((2,6-Dimethoxyphenyl)ethynyl)benzonitrile (1h)}

White solid, White solid, mp: $120-122{ }^{\circ} \mathrm{C} .(88 \%) . \mathrm{R}_{\mathrm{f}}=$ $0.20\left(\mathrm{Et}_{2} \mathrm{O} / \mathrm{Cyclohexane}, 5 / 95, \mathrm{SiO}_{2}\right)$. NMR $\left(\mathrm{CDCl}_{3}, 300\right.$ $\mathrm{MHz}): \delta 3.92(6 \mathrm{H}, \mathrm{s}), 6.57(2 \mathrm{H}, \mathrm{d}, J=8.4 \mathrm{~Hz}), 7.27(1 \mathrm{H}, \mathrm{t}$, $J=8.4 \mathrm{~Hz}), 7.60(2 \mathrm{H}, \mathrm{d}, J=8.6 \mathrm{~Hz}), 7.65(2 \mathrm{H}, \mathrm{d}, J=8.6$ $\mathrm{Hz}) .{ }^{13} \mathrm{C}$ NMR $\left(75 \mathrm{MHz}, \mathrm{CDCl}_{3}\right): \delta 56.2\left(2 \mathrm{CH}_{3}\right), 87.0(\mathrm{C})$, 96.2 (C), 100.7 (C), 103.6 (2CH), 111.0 (C), 118.8 (C), $129.1(\mathrm{C}), 130.9(\mathrm{CH}), 131.9(2 \mathrm{CH}), 132.2(2 \mathrm{CH}), 161.7$ (2C). IR ( $\mathrm{cm}^{-1}$, neat): 2215, 1585, 1475, 1257, 1110, 834, 774, 725. Anal. Calcd for $\mathrm{C}_{17} \mathrm{H}_{13} \mathrm{NO}_{2}$ (263.29): $\mathrm{C} 77.55, \mathrm{H}$ 4.98, found C 77.13, H 4.70.

\section{4-((2,6-Dichlorophenyl)ethynyl)benzonitrile (1i)}

White solid, mp: $144-146{ }^{\circ} \mathrm{C} . \quad(90 \%) . \quad \mathrm{R}_{\mathrm{f}}=0.32$ $\left(\mathrm{Et}_{2} \mathrm{O} / \mathrm{Cyclohexane}, 5 / 95, \mathrm{SiO}_{2}\right)$. NMR $\left(\mathrm{CDCl}_{3}, 300 \mathrm{MHz}\right)$ : $\delta 7.22(1 \mathrm{H}, \mathrm{t}, J=8.8 \mathrm{~Hz}), 7.38(2 \mathrm{H}, \mathrm{d}, J=8.8 \mathrm{~Hz}), 7.66$ $(2 \mathrm{H}, \mathrm{d}, J=8.7 \mathrm{~Hz}), 7.69(2 \mathrm{H}, \mathrm{d}, J=8.7 \mathrm{~Hz})$. NMR $(75$ $\mathrm{MHz}, \mathrm{CDCl}_{3}$ ): $\delta 87.5(\mathrm{C}), 97.7(\mathrm{C}), 112.3(\mathrm{C}), 118.5(\mathrm{C})$, $122.5(\mathrm{C}), 127.5(\mathrm{C}), 127.8(2 \mathrm{CH}), 130.0(\mathrm{CH}), 132.2$ $(2 \mathrm{CH}), 132.4(2 \mathrm{CH}), 137.6(2 \mathrm{C})$. IR ( $\mathrm{cm}^{-1}$, neat): 2229, 1550, 1504, 1425, 1192, 834, 778, 718. Anal. Calcd for $\mathrm{C}_{15} \mathrm{H}_{7} \mathrm{Cl}_{2} \mathrm{~N}$ (272.13): C 66.20, H 2.59, found $\mathrm{C} 65.91, \mathrm{H}$ 2.50 .

\section{1,3-dichloro-2-(phenylethynyl)benzene $(\mathbf{1 j})$}

White solid, $\mathrm{mp}: 56-58{ }^{\circ} \mathrm{C} . \quad(75 \%) . \quad \mathrm{R}_{\mathrm{f}}=0.60$ $\left(\mathrm{Et}_{2} \mathrm{O} / \mathrm{Cyclohexane}, 2 / 98, \mathrm{SiO}_{2}\right)$. NMR $\left(\mathrm{CDCl}_{3}, 300 \mathrm{MHz}\right)$ : $\delta 6.95(1 \mathrm{H}, \mathrm{dd}, J=7.5 \mathrm{~Hz}, J=1.2 \mathrm{~Hz}), 7.10-7.23(5 \mathrm{H}, \mathrm{m})$, 7.35-7.45 (2H, m). ${ }^{13} \mathrm{C}$ NMR (75 MHz, $\left.\mathrm{CDCl}_{3}\right): \delta 83.6(\mathrm{C})$, $100.0(\mathrm{C}), 122.7(\mathrm{C}), 123.5(\mathrm{C}), 127.6(2 \mathrm{CH}), 128.5(2 \mathrm{CH})$, $129.0(\mathrm{CH}), 129.1(\mathrm{CH}), 132.0(2 \mathrm{CH}), 137.3(2 \mathrm{C}) . \mathrm{IR}\left(\mathrm{cm}^{-}\right.$ 1 , neat): 2220, 1553, 1493, 1443, 1428, 1189, 1101, 788, 766, 749, 716. Anal. Calcd for $\mathrm{C}_{14} \mathrm{H}_{8} \mathrm{Cl}_{2}$ (247.12): C 68.04, H 3.26, found C 67.84, H 3.31.

1,3-Dichloro-2-((4-methoxyphenyl)ethynyl)benzene (1k) White solid, mp: 71-73 ${ }^{\circ} \mathrm{C} . \quad(53 \%) . \quad \mathrm{R}_{\mathrm{f}}=0.34$ $\left(\mathrm{Et}_{2} \mathrm{O} / \mathrm{Cyclohexane}, 5 / 95, \mathrm{SiO}_{2}\right) .{ }^{1} \mathrm{H} \mathrm{NMR}\left(\mathrm{CDCl}_{3}, 300\right.$ $\mathrm{MHz}): \delta 3.77(3 \mathrm{H}, \mathrm{s}), 6.83(2 \mathrm{H}, \mathrm{d}, J=8.8 \mathrm{~Hz}), 7.10(1 \mathrm{H}, \mathrm{t}$, 
$J=8.1 \mathrm{~Hz}), 7.26(2 \mathrm{H}, \mathrm{d}, J=8.1 \mathrm{~Hz}), 7.50(2 \mathrm{H}, \mathrm{d}, J=8.8$ $\mathrm{Hz}) .{ }^{13} \mathrm{C}$ NMR $\left(75 \mathrm{MHz}, \mathrm{CDCl}_{3}\right): \delta 55.4\left(\mathrm{CH}_{3}\right), 82.6(\mathrm{C})$, $100.3(\mathrm{C}), 114.2(2 \mathrm{CH}), 114.8(\mathrm{C}), 123.7(\mathrm{C}), 127.6(2 \mathrm{CH})$, $128.6(\mathrm{CH}), 133.5(2 \mathrm{CH}), 137.0(2 \mathrm{C}), 160.3(\mathrm{C})$. IR $\left(\mathrm{cm}^{-1}\right.$, neat): $2195,1604,1509,1429,1248,1024,830,716$. Anal. Calcd for $\mathrm{C}_{15} \mathrm{H}_{10} \mathrm{Cl}_{2} \mathrm{O}$ (277.15): C 65.01, H 3.64, found C 64.88, H 3.54 .

\section{1,3-Dibromo-5-fluoro-2-(phenylethynyl)benzene (11)}

Brown oil. $(55 \%) . \mathrm{R}_{\mathrm{f}}=0.60\left(\mathrm{Et}_{2} \mathrm{O} /\right.$ Cyclohexane, 5/95, $\left.\mathrm{SiO}_{2}\right)$. NMR $\left(\mathrm{CDCl}_{3}, 300 \mathrm{MHz}\right): \delta$ 7.20-7.33 $(3 \mathrm{H}, \mathrm{m}), 7.42$ $-7.48(4 \mathrm{H}, \mathrm{m}) .{ }^{13} \mathrm{C}$ NMR $\left(75 \mathrm{MHz}, \mathrm{CDCl}_{3}\right): \delta_{\mathrm{C}} 73.9(\mathrm{C})$, $81.5(\mathrm{C}), 103.7\left(\mathrm{~d}, J_{C-F}=3.7 \mathrm{~Hz}, \mathrm{C}\right), 119.1\left(\mathrm{~d}, J_{C-F}=24.0\right.$ $\mathrm{Hz}, 2 \mathrm{CH}), 121.7(\mathrm{C}), 129.2(2 \mathrm{CH}), 131.3\left(\mathrm{~d}, J_{C-F}=10.0 \mathrm{~Hz}\right.$, 2C, ), $131.6(\mathrm{CH}), 132.5(2 \mathrm{CH}), 161.9\left(\mathrm{~d}, J_{C-F}=255.0 \mathrm{~Hz}\right.$, C). Anal. Calcd for $\mathrm{C}_{14} \mathrm{H}_{7} \mathrm{Br}_{2} \mathrm{~F}$ (354.01): C 47.50, H 1.99, found C 47.02, H 1.98 .

Methyl 2-( (4-(6-hydroxyhex-1-ynyl)phenyl)ethynyl)-3,4,5trimethoxybenzoate (10) was prepared from methyl-2-iodo3,4,5-trimethoxybenzoate and 6-(4-ethynyl phenyl)hex-5yn-1-ol (in this case heating to $80{ }^{\circ} \mathrm{C}$ was necessary). Yellow solid, mp: $118-120{ }^{\circ} \mathrm{C}$. $(77 \%) . \quad R_{\mathrm{f}}=0.43$ $\left(\mathrm{Et}_{2} \mathrm{O} /\right.$ Cyclohexane, 50/50, $\left.\mathrm{SiO}_{2}\right)$. NMR $\left(\mathrm{CDCl}_{3}, 300\right.$ MHz): $\delta 1.63(4 \mathrm{H}, \mathrm{m}), 2.37(2 \mathrm{H}, \mathrm{t}, J=6.4 \mathrm{~Hz}), 3.59(2 \mathrm{H}, \mathrm{t}$, $J=6.5 \mathrm{~Hz}), 3.79(3 \mathrm{H}, \mathrm{s}), 3.83(3 \mathrm{H}, \mathrm{s}), 3.86(3 \mathrm{H}, \mathrm{s}), 3.92$ $(3 \mathrm{H}, \mathrm{s}), 7,24(1 \mathrm{H}, \mathrm{s}), 7.35(4 \mathrm{H}, \mathrm{m}) .{ }^{13} \mathrm{C}$ NMR $(75 \mathrm{MHz}$, $\left.\mathrm{CDCl}_{3}\right): \delta_{\mathrm{C}} 19.3\left(\mathrm{CH}_{2}\right), 25.0\left(\mathrm{CH}_{2}\right), 31.8\left(\mathrm{CH}_{2}\right), 52.2\left(\mathrm{CH}_{3}\right)$, $56.1\left(\mathrm{CH}_{3}\right), 61.1\left(\mathrm{CH}_{3}\right), 61.3\left(\mathrm{CH}_{3}\right), 62.1\left(\mathrm{CH}_{2}\right), 80.7(\mathrm{C})$, 85.2 (C), 92.1 (C), 97.1 (C), 109.8 (C), 112.1 (C), 122.8 (C), $123.9(\mathrm{C}), 127.6(\mathrm{C}), 131.2(2 \mathrm{CH}), 131.4(2 \mathrm{CH}), 145.7$ (C), 153.0 (C), 155.2 (C), 166.1 (CO). IR ( $\mathrm{cm}^{-1}$, neat): 3058, 2937, 1728, 1338, 1115. Anal. Calcd for $\mathrm{C}_{25} \mathrm{H}_{26} \mathrm{O}_{6}$ (422.47): C 71.07, H 6.20, found C 69.95, H 6.32.

\section{General procedure for the hydrostannation of alkynes (B).}

Tributyltin hydride $(11 \mathrm{mmol})$ was added dropwise at room temperature to a solution of $\mathrm{PdCl}_{2}\left(\mathrm{PPh}_{3}\right)_{2}(0.1 \mathrm{mmol})$ and alkyne $1(10 \mathrm{mmol})$ in THF $(15 \mathrm{~mL})$. The dark brown reaction mixture was stirred for 30 minutes and tributyltin hydride $(2 \mathrm{mmol})$ was added more on the crude mixture to complete the hydrostannation reaction. After stirring for 30 additionnal minutes, the solution was concentrated in vacuo. Purification by flash chromatography on silica gel gave the desirated products.

\section{Hydrostannation of $\mathbf{1 b}$}

(E)-Tributyl(1-(2,6-dimethoxyphenyl)-2-phenylvinyl) stannane (2b)

Colorless oil $(86 \%) . \mathrm{R}_{\mathrm{f}}=0.75\left(\mathrm{Et}_{2} \mathrm{O} /\right.$ Cyclohexane, $1 / 9$ TEA $\left.1 \%, \mathrm{SiO}_{2}\right) .{ }^{1} \mathrm{H}$ NMR $\left(\mathrm{CDCl}_{3}, 300 \mathrm{MHz}\right): \delta 0.73-0.83$ $(15 \mathrm{H}, \mathrm{m}), 1.20(6 \mathrm{H}$, sextet, $J=7.2 \mathrm{~Hz}), 1.32-1.44(6 \mathrm{H}, \mathrm{m})$, $3.57(6 \mathrm{H}, \mathrm{s}), 6.43(2 \mathrm{H}, \mathrm{d}, J=8.3 \mathrm{~Hz}),, 6.64\left(1 \mathrm{H}, \mathrm{s}, J_{\mathrm{Sn}-\mathrm{H}}=\right.$ 67.0 Hz), 6.91-7.11 (6H, m). ${ }^{13} \mathrm{C} \mathrm{NMR}\left(75 \mathrm{MHz}, \mathrm{CDCl}_{3}\right): \delta$ $10.6\left(3 \mathrm{CH}_{2}\right), 13.9\left(3 \mathrm{CH}_{3}\right), 27.5\left(3 \mathrm{CH}_{2}\right), 29.1\left(3 \mathrm{CH}_{2}\right), 55.7$ $\left(2 \mathrm{CH}_{3}\right), 104.0(2 \mathrm{CH}), 122.4(\mathrm{C}), 126.4(\mathrm{CH}), 126.5(\mathrm{CH})$, $127.9(2 \mathrm{CH}), 128.1(2 \mathrm{CH}), 139.2(\mathrm{C}), 139.4(\mathrm{CH}), 141.6$ (C), 155.4 (2C). IR ( $\mathrm{cm}^{-1}$, neat): 2954, 2925, 2870, 2852, 1579, 1494, 1467, 1431, 1375, 1282, 1245, 1171, 1110, 1074, 961, 921, 865, 778, 756, 713. Anal. Calcd for $\mathrm{C}_{28} \mathrm{H}_{42} \mathrm{O}_{2} \mathrm{Sn}$ (529.34): C 63.53, H 8.00, found C 63.41, H 8.05 .

Hydrostannation of 1c

(E)-(1-(2,6-Bis(benzyloxy)phenyl)-2-phenylvinyl)tributylstannane (2c)

Colorless oil $(90 \%) . \mathrm{R}_{\mathrm{f}}=0.56\left(\mathrm{Et}_{2} \mathrm{O} /\right.$ Cyclohexane, $5 / 95$ TEA $\left.1 \%, \mathrm{SiO}_{2}\right) .{ }^{1} \mathrm{H}$ NMR $\left(\mathrm{CDCl}_{3}, 300 \mathrm{MHz}\right): \delta 0.54-0.91$ $(15 \mathrm{H}, \mathrm{m}), 1.10(6 \mathrm{H}$, sextet, $J=7.4 \mathrm{~Hz}), 1.21-1.41(6 \mathrm{H}, \mathrm{m})$, $4.82(2 \mathrm{H}, \mathrm{d}, J=12.4 \mathrm{~Hz}), 4.86(2 \mathrm{H}, \mathrm{d}, J=12.4 \mathrm{~Hz}), 6.43$ $(2 \mathrm{H}, \mathrm{d}, J=8.3 \mathrm{~Hz}), 6.70\left(1 \mathrm{H}, \mathrm{s}, J_{\mathrm{Sn}-\mathrm{H}}=68.0 \mathrm{~Hz}\right), 6.85-7.25$ $(16 \mathrm{H}, \mathrm{m})$. NMR $\left(75 \mathrm{MHz}, \mathrm{CDCl}_{3}\right): \delta 10.7\left(3 \mathrm{CH}_{2}\right), 13.8$ $\left(3 \mathrm{CH}_{3}\right), 27.5\left(3 \mathrm{CH}_{2}\right), 29.0\left(3 \mathrm{CH}_{2}\right), 70.1\left(2 \mathrm{CH}_{2}\right), 105.6$ $(2 \mathrm{CH}), 123.5(\mathrm{C}), 126.3(\mathrm{CH}), 126.4(\mathrm{CH}), 127.0(4 \mathrm{CH})$, $127.5(2 \mathrm{CH}), 128.0(2 \mathrm{CH}), 128.2(2 \mathrm{CH}), 128.3(4 \mathrm{CH})$, 137.7 (2C), 139.3 (C), 139.5 (1CH), 142.0 (C), 154.5 (2C). IR ( $\mathrm{cm}^{-1}$, neat): 2954, 2921, 2870, 2852, 1580, 1496, 1449 , $1418,1377,1246,1229,1201,1177,1155,1096,1074$, 1029, 961, 922, 864, 843, 733, 693. Anal. Calcd for $\mathrm{C}_{40} \mathrm{H}_{50} \mathrm{O}_{2} \mathrm{Sn}$ (681.53): C 70.49, H 7.39, found C $70.10 \mathrm{H}$ 7.50 .

Hydrostannation of 1d

(E)-2-(2-Phenyl-1-(tributylstannyl)vinyl)-1,3-phenylene diacetate (2d)

Colorless oil $(65 \%) ; \mathrm{R}_{\mathrm{f}}=0.60\left(\mathrm{Et}_{2} \mathrm{O} /\right.$ Cyclohexane, $3 / 7$ TEA $\left.1 \%, \mathrm{SiO}_{2}\right) .{ }^{1} \mathrm{H}$ NMR $\left(\mathrm{CDCl}_{3}, 300 \mathrm{MHz}\right): \delta 0.63-1.10$ $(15 \mathrm{H}, \mathrm{m}), 1.23(6 \mathrm{H}$, sextet, $J=7.4 \mathrm{~Hz}), 1.33-1.58(6 \mathrm{H}, \mathrm{m})$, $2.02(6 \mathrm{H}, \mathrm{s}), 6.66\left(1 \mathrm{H}, \mathrm{s}, J_{\mathrm{Sn}-\mathrm{H}}=61.4 \mathrm{~Hz}\right), 6.87(2 \mathrm{H}, \mathrm{d}, J=$ 8.0 Hz), 6.98-7.21 (6H, m). ${ }^{13} \mathrm{C} \mathrm{NMR}\left(75 \mathrm{MHz}, \mathrm{CDCl}_{3}\right): \delta$ $11.3\left(3 \mathrm{CH}_{2}\right), 13.8\left(3 \mathrm{CH}_{3}\right), 21.0\left(2 \mathrm{CH}_{3}\right), 27.6\left(3 \mathrm{CH}_{2}\right), 29.0$ $\left(3 \mathrm{CH}_{2}\right), \quad 120.3(2 \mathrm{CH}), \quad 126.2(\mathrm{CH}), 127.4(\mathrm{CH}), 128.2$ $(2 \mathrm{CH}), 128.3(2 \mathrm{CH}), 131.8(\mathrm{C}), 137.6(\mathrm{C}), 139.3(\mathrm{C})$, $142.0(1 \mathrm{CH}), 146.9(2 \mathrm{C}), 168.8(2 \mathrm{CO}) . \mathrm{IR}\left(\mathrm{cm}^{-1}\right.$, neat): 2956, 2921, 2851, 1767, 1493, 1455, 1366, 1184, 1156, 1074, 1025, 985, 962, 926, 881, 867. Anal. Calcd for $\mathrm{C}_{30} \mathrm{H}_{42} \mathrm{O}_{4} \mathrm{Sn}$ (585.36): C 61.56, H 7.23, found C 61.74, H 7.48 .

Hydrostannation of $\mathbf{1 e}$

(E)-Tributyl(1-(2,6-dimethoxyphenyl)-2-(4-methoxyphenyl) vinyl)stannane (2e)

Colorless oil $(71 \%) . \mathrm{R}_{\mathrm{f}}=0.14\left(\mathrm{Et}_{2} \mathrm{O} /\right.$ Cyclohexane, $2 / 98$ TEA $\left.1 \%, \mathrm{SiO}_{2}\right) .{ }^{1} \mathrm{H}$ NMR $\left(\mathrm{CDCl}_{3}, 300 \mathrm{MHz}\right): \delta 0.66-0.92$ $(15 \mathrm{H}, \mathrm{m}, 1.20(6 \mathrm{H}$, sextet, $J=7.3 \mathrm{~Hz}), 1.30-1.44(6 \mathrm{H}, \mathrm{m})$, $3.57(6 \mathrm{H}, \mathrm{s}), 3.65(3 \mathrm{H}, \mathrm{s}), 6.44(2 \mathrm{H}, \mathrm{d}, J=8.3 \mathrm{~Hz}), 6.52-$ $6.67(3 \mathrm{H}, \mathrm{m}), 6.93(2 \mathrm{H}, \mathrm{d}, J=8.8 \mathrm{~Hz}), 7.05(1 \mathrm{H}, \mathrm{t}, J=8.3$ $\mathrm{Hz}) .{ }^{13} \mathrm{C}$ NMR $\left(75 \mathrm{MHz}, \mathrm{CDCl}_{3}\right): \delta 10.6\left(3 \mathrm{CH}_{2}\right), 13.9$ $\left(3 \mathrm{CH}_{3}\right), 27.5\left(3 \mathrm{CH}_{2}\right), 29.1\left(3 \mathrm{CH}_{2}\right), \quad 55.3\left(\mathrm{CH}_{3}\right), 55.7$ $\left(2 \mathrm{CH}_{3}\right), 104.1(2 \mathrm{CH}), 113.3(2 \mathrm{CH}), 126.4(\mathrm{CH}), 129.4$ (2CH), $132.3(\mathrm{C}), 138.6(\mathrm{CH}), 138.8(\mathrm{C}), 155.5(2 \mathrm{C}), 158.2$ 
(C), 177.1 (C). IR ( $\mathrm{cm}^{-1}$, neat): 2994, 2954, 2927, 2870, 2835, 1604, 1582, 1508, 1466, 1431, 1375, 1292, 1244, 1175 1109, 1036, 960, 896, 874, 823, 805, 781, 760, 732. Anal. Calcd for $\mathrm{C}_{29} \mathrm{H}_{44} \mathrm{O}_{3} \mathrm{Sn}$ (559.37): C 62.27, H 7.93, found C 62.00, H 8.18.

Hydrostannation of $\mathbf{1 f}$

(E)-(1-(2,6-Bis(benzyloxy)phenyl)-2-(4-methoxyphenyl)vinyl)tributylstannane $(\mathbf{2 f})$

Colorless oil $(55 \%) . \mathrm{R}_{\mathrm{f}}=0.37\left(\mathrm{Et}_{2} \mathrm{O} /\right.$ Cyclohexane, 5/95 TEA $\left.1 \%, \mathrm{SiO}_{2}\right) .{ }^{1} \mathrm{H}$ NMR $\left(\mathrm{CDCl}_{3}, 300 \mathrm{MHz}\right): \delta 0.70-0.92$ $(15 \mathrm{H}, \mathrm{m}), 1.18$ (6H, sextet, $J=7.3 \mathrm{~Hz}), 1.29-1.49(6 \mathrm{H}, \mathrm{m})$, $3.75(3 \mathrm{H}, \mathrm{s}), 4.94(2 \mathrm{H}, \mathrm{d}, J=12.7 \mathrm{~Hz}), 4.98(2 \mathrm{H}, \mathrm{d}, J=$ $12.7 \mathrm{~Hz}), 6.53(2 \mathrm{H}, \mathrm{d}, J=8.3 \mathrm{~Hz}), 6.67(2 \mathrm{H}, \mathrm{d}, J=8.6 \mathrm{~Hz})$, $6.73\left(1 \mathrm{H}, \mathrm{s}, J_{\mathrm{Sn}-\mathrm{H}}=67.0 \mathrm{~Hz}\right), 6.97-7.10(3 \mathrm{H}, \mathrm{m}), 7.17-7.38$ $(10 \mathrm{H}, \mathrm{m}) .{ }^{13} \mathrm{C} \mathrm{NMR}\left(75 \mathrm{MHz}, \mathrm{CDCl}_{3}\right): \delta_{\mathrm{C}} 10.6\left(3 \mathrm{CH}_{2}\right)$, $13.8\left(3 \mathrm{CH}_{3}\right), 27.5\left(3 \mathrm{CH}_{2}\right), 29.0\left(3 \mathrm{CH}_{2}\right), 55.2\left(\mathrm{CH}_{3}\right), 70.1$ $\left(2 \mathrm{CH}_{2}\right), 105.8(2 \mathrm{CH}), 113.4(2 \mathrm{CH}), 123.8(\mathrm{C}), 126.2(\mathrm{CH})$, $127.0(4 \mathrm{CH}), 127.4(2 \mathrm{CH}), 128.3(4 \mathrm{CH}), 129.4(2 \mathrm{CH})$, 132.5 (C), 137.8 (2C), $138.8(\mathrm{CH}), 139.3(\mathrm{C}), 154.7$ (2C), 158.2 (C). IR (cm ${ }^{-1}$, neat): 2953, 2929, 2869, 2852, 2836, 1604, 1582, 1508, 1464 1449, 1417, 1377, 1291, 1245, 1226, 1201, 1175, 1110, 1095, 1072, 1031, 959, 935, 901, 877, 862, 823, 784, 766. Anal. Calcd for $\mathrm{C}_{41} \mathrm{H}_{52} \mathrm{O}_{3} \mathrm{Sn}$ (711.56): C 69.21, H 7.37, found C 69.36, H 7.20.

Hydrostannation of $\mathbf{1 g}$

(E)-2-(2-(4-Methoxyphenyl)-1-(tributylstannyl)vinyl)-1,3phenylene diacetate $(\mathbf{2 g})$

Colorless oil $(70 \%) . \mathrm{R}_{\mathrm{f}}=0.52\left(\mathrm{Et}_{2} \mathrm{O} /\right.$ Cyclohexane, 30/70 TEA $\left.1 \%, \mathrm{SiO}_{2}\right) .{ }^{1} \mathrm{H}$ NMR $\left(\mathrm{CDCl}_{3}, 300 \mathrm{MHz}\right): \delta 0.64-1.00$ $(15 \mathrm{H}, \mathrm{m}), 1.22(6 \mathrm{H}$, sextet, $J=7.3 \mathrm{~Hz}), 1.34-1.49(6 \mathrm{H}, \mathrm{m})$, $2.02(6 \mathrm{H}, \mathrm{s}), 3.65(3 \mathrm{H}, \mathrm{s}), 6.58\left(1 \mathrm{H}, \mathrm{s}, J_{\mathrm{Sn}-\mathrm{H}}=62.0 \mathrm{~Hz}\right)$, $6.61(2 \mathrm{H}, \mathrm{d}, J=8.8 \mathrm{~Hz}), 6.88(2 \mathrm{H}, \mathrm{d}, J=8.1 \mathrm{~Hz}), 6.96(2 \mathrm{H}$, $\mathrm{d}, J=8.8 \mathrm{~Hz}), 7.13(1 \mathrm{H}, \mathrm{t}, J=8.1 \mathrm{~Hz}) .{ }^{13} \mathrm{C} \mathrm{NMR}(75 \mathrm{MHz}$, $\left.\mathrm{CDCl}_{3}\right): \delta 11.3\left(3 \mathrm{CH}_{2}\right), 13.8\left(3 \mathrm{CH}_{3}\right), 21.0\left(2 \mathrm{CH}_{3}\right), 27.6$ $\left(3 \mathrm{CH}_{2}\right), 29.0\left(3 \mathrm{CH}_{2}\right), 55.2\left(\mathrm{CH}_{3}\right), 113.5(2 \mathrm{CH}), 120.3$ $(2 \mathrm{CH}), 126.0(\mathrm{CH}), 129.7(2 \mathrm{CH}), 130.6(\mathrm{C}), 132.1(\mathrm{C})$, 136.2 (C), $141.3(\mathrm{CH}), 147.2$ (2C), 158.9 (C), 168.9 (2CO). IR ( $\mathrm{cm}^{-1}$, neat): 2955, 2929, 1767, 1604, 1509, 14541419 , $1367,1293,1250,1191,1177,1157,1113,1074,1026$, 985, 959, 908, 878, 825, 802, 781. Anal. Calcd for $\mathrm{C}_{31} \mathrm{H}_{44} \mathrm{O}_{5} \mathrm{Sn}$ (615.39): C 60.50, H 7.21, found $\mathrm{C} 60.24, \mathrm{H}$ 7.18 .

Hydrostannation of $\mathbf{1 h}$

(E)-4-(2-(2,6-dimethoxyphenyl)-2-(tributylstannyl)vinyl) benzonitrile (2l) (major $\alpha$-isomer)

Colorless oil $(86 \%) . \mathrm{R}_{\mathrm{f}}=0.59\left(\mathrm{Et}_{2} \mathrm{O} /\right.$ Cyclohexane, $30 / 70$ TEA $\left.1 \%, \mathrm{SiO}_{2}\right) .{ }^{1} \mathrm{H} \mathrm{NMR}\left(\mathrm{CDCl}_{3}, 300 \mathrm{MHz}\right): \delta 0.73-1.01$ $(15 \mathrm{H}, \mathrm{m}), 1.27(6 \mathrm{H}$, sextet, $J=7.4 \mathrm{~Hz}), 1.37-1.53(6 \mathrm{H}, \mathrm{m})$, $3.61(6 \mathrm{H}, \mathrm{s}), 6.50(2 \mathrm{H}, \mathrm{d}, J=8.4 \mathrm{~Hz}), 6.72\left(1 \mathrm{H}, \mathrm{s},{ }^{3} J_{\mathrm{Sn}-\mathrm{H}}=\right.$ $66.0 \mathrm{~Hz}), 7.05-7.20(3 \mathrm{H}, \mathrm{m}), 7.37(2 \mathrm{H}, \mathrm{d}, J=8.4 \mathrm{~Hz}) .{ }^{13} \mathrm{C}$ NMR $\left(75 \mathrm{MHz}, \mathrm{CDCl}_{3}\right): \delta 10.7\left(3 \mathrm{CH}_{2}\right), 13.9\left(3 \mathrm{CH}_{3}\right), 27.5$ $\left(3 \mathrm{CH}_{2}\right), 29.0\left(3 \mathrm{CH}_{2}\right), 55.6\left(2 \mathrm{CH}_{3}\right), 103.9(2 \mathrm{CH}), 109.4(\mathrm{C})$, $119.5(\mathrm{CN}), 121.2(\mathrm{C}), 127.3(\mathrm{CH}), 128.4(2 \mathrm{CH}), 131.8$
(2CH), $137.6(\mathrm{CH}), 143.5$ (C), 147.8 (C), 155.0 (2C). IR $\left(\mathrm{cm}^{-1}\right.$, neat): 2957, 2925, 2893, 2870, 2853, 2836, 2226, $1730,1714,1600,1582,1494,1468,1431,1376,1290$, 1246, 1208, 1173, 1155, 1111, 1075, 1039, 960, 901, 879, 862, 840, 820, 781, 757. Anal. Calcd for $\mathrm{C}_{29} \mathrm{H}_{41} \mathrm{NO}_{2} \mathrm{Sn}$ (554.35): C 62.83, H 7.45, found C 62.75, H 7.27.

\section{Hydrostannation of $\mathbf{1 i}$ \\ (E)-4-(2-(2,6-Dichlorophenyl)-2-(tributylstannyl)vinyl) benzonitrile (2i)}

Colorless oil $(88 \%) . \mathrm{R}_{\mathrm{f}}=0.50\left(\mathrm{Et}_{2} \mathrm{O} /\right.$ Cyclohexane, 5/95 TEA $\left.1 \%, \mathrm{SiO}_{2}\right) .{ }^{1} \mathrm{H} \mathrm{NMR}\left(\mathrm{CDCl}_{3}, 300 \mathrm{MHz}\right): \delta 0.79(9 \mathrm{H}, \mathrm{t}$, $J=7.3 \mathrm{~Hz}), 0.86-1.00(6 \mathrm{H}, \mathrm{m}), 1.21(6 \mathrm{H}$, sextet, $J=7.3$ $\mathrm{Hz}), 1.33-1.48(6 \mathrm{H}, \mathrm{m}), 6.73\left(1 \mathrm{H}, \mathrm{s}, J_{\mathrm{Sn}-\mathrm{H}}=57.0 \mathrm{~Hz}\right), 6.92-$ $7.08(3 \mathrm{H}, \mathrm{m}), 7.21(2 \mathrm{H}, \mathrm{d}, J=8.0 \mathrm{~Hz}), 7.36(2 \mathrm{H}, \mathrm{d}, J=8.4$ $\mathrm{Hz}) .{ }^{13} \mathrm{C} \mathrm{NMR}\left(75 \mathrm{MHz}, \mathrm{CDCl}_{3}\right): \delta 11.5\left(3 \mathrm{CH}_{2}\right), 13.7$ $\left(3 \mathrm{CH}_{3}\right), 27.4\left(3 \mathrm{CH}_{2}\right), 28.8\left(3 \mathrm{CH}_{2}\right), 110.5(\mathrm{C}), 119.1(\mathrm{CN})$, $127.3(\mathrm{CH}), 128.2(2 \mathrm{CH}), 128.4(2 \mathrm{CH}), 131.4(2 \mathrm{C}), 132.2$ (2CH), $138.5(\mathrm{CH}), 141.8(\mathrm{C}), 142.2$ (C), 150.5 (C). IR ( $\mathrm{cm}^{-1}$, neat): 2955, 2930, 2871, 2851, 2227, 1602, 1553, $1502,1462,1426,1405,1377,1339,1253,1187,1148$, 1088, 1071, 1017, 961, 903, 876, 824, 777, 729. Anal. Calcd for $\mathrm{C}_{27} \mathrm{H}_{35} \mathrm{Cl}_{2} \mathrm{NSn}$ (563.19): C 57.58, H 6.26, found C 57.41, H 6.03 .

Hydrostannation of $\mathbf{1 j}$

(E)-Tributyl (1- (2,6-dichlorophenyl)-2-phenylvinyl) stannane $(\mathbf{2} \mathbf{j})$

Colorless oil $(98 \%) . \mathrm{R}_{\mathrm{f}}=0.64\left(\mathrm{Et}_{2} \mathrm{O} /\right.$ Cyclohexane, $2 / 98$ TEA $\left.1 \%, \mathrm{SiO}_{2}\right) .{ }^{1} \mathrm{H} \mathrm{NMR}\left(\mathrm{CDCl}_{3}, 300 \mathrm{MHz}\right): \delta 0.79(9 \mathrm{H}, \mathrm{t}$, $J=7.3 \mathrm{~Hz}), 0.85-1.01(6 \mathrm{H}, \mathrm{m}), 1.21(6 \mathrm{H}$, sextet, $J=7.3$ $\mathrm{Hz}), 1.32-1.47(6 \mathrm{H}, \mathrm{m}), 6.70\left(1 \mathrm{H}, \mathrm{s}, J_{\mathrm{Sn}-\mathrm{H}}=60.8 \mathrm{~Hz}\right), 6.88-$ $7.14(6 \mathrm{H}, \mathrm{m}), 7.21(2 \mathrm{H}, \mathrm{d}, J=7.9 \mathrm{~Hz}) .{ }^{13} \mathrm{C} \mathrm{NMR}(75 \mathrm{MHz}$, $\left.\mathrm{CDCl}_{3}\right): \delta 11.3\left(3 \mathrm{CH}_{2}\right), 13.8\left(3 \mathrm{CH}_{3}\right), 27.5\left(3 \mathrm{CH}_{2}\right), 28.9$ $\left(3 \mathrm{CH}_{2}\right), 126.8(\mathrm{CH}), 127.3(\mathrm{CH}), 127.9(2 \mathrm{CH}), 128.0$ (2CH), $128.3(2 \mathrm{CH}), 131.8(2 \mathrm{C}), 138.0(\mathrm{C}), 140.3(1 \mathrm{CH})$, 142.8 (C), 144.8 (C). IR ( $\mathrm{cm}^{-1}$, neat): 2955, 2923, 2870, 2851, 1553, 1493, 1463, 1425, 1376, 1254, 1191, 1148, 1088, 1073, 1047, 1028, 1001, 960, 921, 893, 874, 774. Anal. Calcd for $\mathrm{C}_{26} \mathrm{H}_{36} \mathrm{Cl}_{2} \mathrm{Sn}$ (538.18): C 58.02, H 6.74, found C 58.37, H 6.77.

\section{Hydrostannation of $\mathbf{1 k}$}

(E)-Tributyl(1-(2,6-dichlorophenyl)-2-(4-methoxyphenyl)vinyl)stannane (2k)

Colorless oil $(98 \%) . \mathrm{R}_{\mathrm{f}}=0.43\left(\mathrm{Et}_{2} \mathrm{O} /\right.$ Cyclohexane, 5/95 TEA $\left.1 \%, \mathrm{SiO}_{2}\right) .{ }^{1} \mathrm{H} \mathrm{NMR}\left(\mathrm{CDCl}_{3}, 300 \mathrm{MHz}\right): \delta 0.80(9 \mathrm{H}, \mathrm{t}$, $J=7.3 \mathrm{~Hz}), 0.84-0.95(6 \mathrm{H}, \mathrm{m}), 1.20(6 \mathrm{H}$, sextet, $J=7.3$ $\mathrm{Hz}), 1.33-1.53(6 \mathrm{H}, \mathrm{m}), 6.60(2 \mathrm{H}, \mathrm{d}, J=8.8 \mathrm{~Hz}), 3.66(3 \mathrm{H}$, s), $6.62\left(1 \mathrm{H}, \mathrm{s}, J_{\mathrm{Sn}-\mathrm{H}}=62.1 .0 \mathrm{~Hz}\right), 6.84(2 \mathrm{H}, \mathrm{d}, J=8.8 \mathrm{~Hz})$, $6.95(1 \mathrm{H}, \mathrm{t}, J=8.3 \mathrm{~Hz}), 7.21(2 \mathrm{H}, \mathrm{d}, J=8.0 \mathrm{~Hz}) .{ }^{13} \mathrm{C} \mathrm{NMR}$ $\left(75 \mathrm{MHz}, \mathrm{CDCl}_{3}\right): \delta 11.3\left(3 \mathrm{CH}_{2}\right), 13.8\left(3 \mathrm{CH}_{3}\right), 27.5$ $\left(3 \mathrm{CH}_{2}\right), 28.9\left(3 \mathrm{CH}_{2}\right), 55.3\left(1 \mathrm{CH}_{3}\right), 113.8(2 \mathrm{CH}), 126.7$ $(\mathrm{CH}), 128.0(2 \mathrm{CH}), 129.4(2 \mathrm{CH}), 131.0(\mathrm{C}), 132.1(2 \mathrm{C})$, $139.7(\mathrm{CH}), 142.0(\mathrm{C}), 142.9$ (C), 158.8 (C). IR (cm-1, neat): 2955, 2925, 2870, 2852, 1603, 1552, 1508, 1462, 
1426, 1376, 1340, 1292, 1249, 1176, 1148, 1112, 1088, 1072, 1036, 960, 879, 823. Anal. Calcd for $\mathrm{C}_{27} \mathrm{H}_{38} \mathrm{Cl}_{2} \mathrm{OSn}$ (568.21): C 57.07, H 6.74, found C 57.22, H 6.38.

Hydrostannation of $\mathbf{1 l}$

(E)-Tributyl(1-(2,6-dibromo-4-fluorophenyl)-2-phenylvin yl) stannane (2l)

Colorless oil $(48 \%) . \mathrm{R}_{\mathrm{f}}=0.50\left(\mathrm{Et}_{2} \mathrm{O} /\right.$ Cyclohexane, $2 / 98$ TEA $\left.1 \%, \mathrm{SiO}_{2}\right) .{ }^{1} \mathrm{H}$ NMR $\left(\mathrm{CDCl}_{3}, 300 \mathrm{MHz}\right): \delta 0.54-0.86$ $(15 \mathrm{H}, \mathrm{m}), 1.08-1.42(12 \mathrm{H}, \mathrm{m}), 6.29\left(1 \mathrm{H}, \mathrm{s}, J_{\mathrm{Sn}-\mathrm{H}}=66.0 \mathrm{~Hz}\right)$, 7.05-7.10 (1H, m), $7.18(2 \mathrm{H}, \mathrm{t}, J=6.9 \mathrm{~Hz}), 7.29(2 \mathrm{H}, \mathrm{d}, J=$ $7.6 \mathrm{~Hz}), 7.37(2 \mathrm{H}, \mathrm{m}) .{ }^{13} \mathrm{C}$ NMR $\left(75 \mathrm{MHz}, \mathrm{CDCl}_{3}\right): \delta 11.0$ $\left(3 \mathrm{CH}_{2}\right), 13.7\left(3 \mathrm{CH}_{3}\right), 27.5\left(3 \mathrm{CH}_{2}\right), 29.0\left(3 \mathrm{CH}_{2}\right), 103.7(\mathrm{~d}, J$ C-F $=5.0 \mathrm{~Hz}, \mathrm{C}), 119.1\left(\mathrm{~d}, J_{\mathrm{C}-\mathrm{F}}=24.0 \mathrm{~Hz}, 2 \mathrm{CH}\right), 126.4(\mathrm{C})$, $128.1(2 \mathrm{CH}), 128.2(2 \mathrm{CH}), 131.3\left(\mathrm{~d}, J_{\mathrm{C}-\mathrm{F}}=10.0 \mathrm{~Hz}, 2 \mathrm{C}\right)$, $131.6(\mathrm{CH}), 140.1(1 \mathrm{C}), 154.2(\mathrm{CH}), 161.9\left(\mathrm{~d},{ }^{1} J_{\mathrm{C}-\mathrm{F}}=\right.$ $255.0 \mathrm{~Hz}, \mathrm{C}$ ). Anal. Calcd for $\mathrm{C}_{26} \mathrm{H}_{35} \mathrm{Br}_{2} \mathrm{FSn}$ (645.07): $\mathrm{C}$ 48.41, H 5.47, found C 48.60, H 5.33.

Hydrostannation of $\mathbf{1 n}$

(E)-3-Mesityl-3-(tributylstannyl)prop-2-en-1-ol (2n)

Colorless oil $(81 \%) . \mathrm{R}_{\mathrm{f}}=0.41(\mathrm{AcOEt} /$ Cyclohexane, $5 / 95$ TEA $\left.1 \%, \mathrm{SiO}_{2}\right) .{ }^{1} \mathrm{H}$ NMR $\left(\mathrm{CDCl}_{3}, 300 \mathrm{MHz}\right): \delta 0.78-0.86$ $(9 \mathrm{H}, \mathrm{m}), 1.16-1.44(19 \mathrm{H}, \mathrm{m}), 2.05(6 \mathrm{H}, \mathrm{s}), 2.23(3 \mathrm{H}, \mathrm{s})$, $3.87(2 \mathrm{H}, \mathrm{s}), 5.98\left(1 \mathrm{H}, \mathrm{t}, J=5.6 \mathrm{~Hz}, J_{\mathrm{Sn}-\mathrm{H}}=63.0 \mathrm{~Hz}\right), 6.78$ $(2 \mathrm{H}, \mathrm{s}) .{ }^{13} \mathrm{C}$ NMR $\left(75 \mathrm{MHz}, \mathrm{CDCl}_{3}\right): \delta 10.9\left(3 \mathrm{CH}_{2}\right), 14.1$ $\left(3 \mathrm{CH}_{3}\right), 20.9\left(2 \mathrm{CH}_{3}\right), 21.3\left(\mathrm{CH}_{3}\right), 27.8\left(3 \mathrm{CH}_{2}\right), 29.2$ $\left(3 \mathrm{CH}_{2}\right), 61.8\left(\mathrm{CH}_{2}\right), 128.4(2 \mathrm{CH}), 132.8(\mathrm{C}), 134.8(\mathrm{C})$, 139.7 (C), $139.8(2 \mathrm{C}), 148.4(\mathrm{CH})$. IR $\left(\mathrm{cm}^{-1}\right.$, neat): 3340 , 2950, 2930, 2840, 1460, 1370, 1010. Anal. Calcd for $\mathrm{C}_{24} \mathrm{H}_{42} \mathrm{OSn}$ (465.30): C 61.95, H 9.10, found C 62.21, H 8.98 .

Hydrostannation of $\mathbf{1 0}$

Methyl-2-((E)-2-(4-( (E)-6-hydroxy-1-(tributyl stannyl) hex-1-enyl)phenyl)-1-(tributylstannyl)vinyl)-3,4,5 trimethoxybenzoate (2o)

Yellow oil $(91 \%) . \mathrm{R}_{\mathrm{f}}=0.28($ AcOEt/Cyclohexane, 30/70 TEA $\left.1 \%, \mathrm{SiO}_{2}\right) .{ }^{1} \mathrm{H}$ NMR $\left(\mathrm{CDCl}_{3}, 300 \mathrm{MHz}\right): \delta 0.35(30 \mathrm{H}$, $\mathrm{m}), 1.23(14 \mathrm{H}, \mathrm{m}), 1.43(14 \mathrm{H}, \mathrm{m}), 1.97(2 \mathrm{H}, \mathrm{q}, J=7.0 \mathrm{~Hz})$, $3.48(2 \mathrm{H}, \mathrm{dd}, J=5.5 \mathrm{~Hz}), 3.57(3 \mathrm{H}, \mathrm{s}), 3.65(3 \mathrm{H}, \mathrm{s}), 3.89$ $(3 \mathrm{H}, \mathrm{s}), 3.90(3 \mathrm{H}, \mathrm{s}), 5.66\left(1 \mathrm{H}, \mathrm{t}, J=7.0 \mathrm{~Hz},{ }^{3} J_{S n-H}=62.0\right.$ $\mathrm{Hz}), 6.61\left(1 \mathrm{H}, \mathrm{s}, J_{S n-H}=66.0 \mathrm{~Hz}\right), 6.64(2 \mathrm{H}, \mathrm{d}, J=8.2 \mathrm{~Hz})$, $6.81(2 \mathrm{H}, \mathrm{d}, J=8.2 \mathrm{~Hz}), 7.23(1 \mathrm{H}, \mathrm{s}) .{ }^{13} \mathrm{C}$ NMR $(75 \mathrm{MHz}$, $\left.\mathrm{CDCl}_{3}\right): \delta 10.9\left(6 \mathrm{CH}_{2}\right), 13.6\left(6 \mathrm{CH}_{3}\right), 25.7\left(\mathrm{CH}_{2}\right), 26.9$ $\left(\mathrm{CH}_{2}\right), 27.2\left(6 \mathrm{CH}_{2}\right), 28.9\left(6 \mathrm{CH}_{2}\right), 31.9\left(\mathrm{CH}_{2}\right), 51.7\left(\mathrm{CH}_{3}\right)$, $55.9\left(\mathrm{CH}_{3}\right), 60.6\left(\mathrm{CH}_{3}\right), 60.7\left(\mathrm{CH}_{3}\right), 62.4\left(\mathrm{CH}_{2}\right), 109.7$ $(\mathrm{CH}), 122.5(\mathrm{C}), 126.7(2 \mathrm{CH}), 127.9(2 \mathrm{CH}), 134.7(\mathrm{C})$, $135.4(\mathrm{C}), 137.2(\mathrm{CH}), 141.6(\mathrm{CH}), 143.4(\mathrm{C}), 144.8(\mathrm{C})$, 145.3 (C), 146.1 (C), 148.9 (C), 150.8 (C), 167,7 (C). IR ( $\mathrm{cm}^{-1}$, neat): 2961, 2849, 1721, 1589, 1291, 1061. Anal. Calcd for $\mathrm{C}_{49} \mathrm{H}_{82} \mathrm{O}_{6} \mathrm{Sn}_{2}$ (1004.59): C 58.58, H 8.23, found C 58.39, H 8.50.

Hydrostannation of $\mathbf{1 p}$

Tributyl(1-(2,6-dimethoxyphenyl)vinyl)stannane (2p)
Colorless oil (92\%). $\mathrm{R}_{\mathrm{f}}=0.70(\mathrm{AcOEt} /$ Cyclohexane, $5 / 95$ TEA $\left.1 \%, \mathrm{SiO}_{2}\right) .{ }^{1} \mathrm{H}$ NMR $\left(300 \mathrm{MHz}, \mathrm{CDCl}_{3}\right) \delta$ 0.93-0.74 $(15 \mathrm{H}, \mathrm{m}), 1.62-1.09(12 \mathrm{H}, \mathrm{m}), 3.75(6 \mathrm{H}, \mathrm{s}), 5.63(1 \mathrm{H}, \mathrm{d}, J$ $\left.=3.3 \mathrm{~Hz}, J_{\mathrm{H}-\mathrm{Sn}}=64.3 \mathrm{~Hz}\right), 5.89\left(1 \mathrm{H}, \mathrm{d}, J=3.3 \mathrm{~Hz}, J_{\mathrm{H}-\mathrm{Sn}}=\right.$ $137.0 \mathrm{~Hz}), 6.52(2 \mathrm{H}, \mathrm{t}, J=8.5 \mathrm{~Hz}), 7.08(1 \mathrm{H}, \mathrm{t}, J=8.5 \mathrm{~Hz})$. ${ }^{13} \mathrm{C} \mathrm{NMR}\left(75 \mathrm{MHz}, \mathrm{CDCl}_{3}\right) \delta 10.6\left(3 \mathrm{CH}_{2}\right), 13.9\left(3 \mathrm{CH}_{3}\right)$, $27.5\left(3 \mathrm{CH}_{2}\right), 29.1\left(3 \mathrm{CH}_{2}\right), 55.80\left(2 \mathrm{CH}_{3}\right), 103.9(2 \mathrm{CH})$, 124.2 (C), $126.4(\mathrm{CH}), 129.0$ (2C), $147.4(\mathrm{C}), 156.0(\mathrm{C})$. IR ( $\mathrm{cm}^{-1}$, neat): 2957, 2928, 2870, 1585, 1468, 1433, 1247, 1115. Anal. Calcd for $\mathrm{C}_{22} \mathrm{H}_{38} \mathrm{O}_{2} \mathrm{Sn}$ (453.25): C 58.30, H 8.45, found C 58.34, H 8.42.

\section{Hydrostannation of $\mathbf{1 q}$}

Tributyl(1-(2,6-dichlorophenyl)vinyl)stannane (2q)

Colorless oil $(98 \%) . \mathrm{R}_{\mathrm{f}}=0.8$ (Cyclohexane, TEA $1 \%$, $\left.\mathrm{SiO}_{2}\right) .{ }^{1} \mathrm{H}$ NMR (300 MHz, $\left.\mathrm{CDCl}_{3}\right) \delta$ 1.03-0.74 (15H, m), $1.52-1.15(12 \mathrm{H}, \mathrm{m}), 5.69(1 \mathrm{H}, \mathrm{d}, J=2.5 \mathrm{~Hz}), 5.79(1 \mathrm{H}, \mathrm{d}, J$ $=2.5 \mathrm{~Hz}), 7.01(1 \mathrm{H}, \mathrm{t}, J=8.0 \mathrm{~Hz}), 7.39-7.17(2 \mathrm{H}, \mathrm{m}) \cdot{ }^{13} \mathrm{C}$ NMR $\left(75 \mathrm{MHz}, \mathrm{CDCl}_{3}\right) \delta 11.3\left(3 \mathrm{CH}_{2}\right), 13.8\left(3 \mathrm{CH}_{3}\right), 27.4$ $\left(3 \mathrm{CH}_{2}\right), 28.9\left(3 \mathrm{CH}_{2}\right), 126.8(\mathrm{CH}), 127.8(2 \mathrm{CH}), 130.1(2 \mathrm{C})$, 132.2 (C), 152.2 (C). IR ( $\mathrm{cm}^{-1}$, neat): 2955, 2922, 1555, 1425, 1192, 1075, 930, 774, 743, 688. Anal. Calcd for $\mathrm{C}_{20} \mathrm{H}_{32} \mathrm{Cl}_{2} \mathrm{Sn}$ (462.08): C 51.98, H 6.98, found C $51.95, \mathrm{H}$ 6.94 .

\section{Typical Procedure for Iodination of Ortho-Ortho' Vinylstannes (C).}

To a flame-dried flask under a nitrogen atmosphere were added at $0^{\circ} \mathrm{C}$ vinyl stannane $2 \mathbf{k}$ ( 1 equiv.), dry $\mathrm{CH}_{2} \mathrm{Cl}_{2}(2$ $\mathrm{mL}$ per mmol of substrate), sublimed finely divided $\mathrm{I}_{2}$ (1.05 equiv.) and the dark wine solution was vigorously stirred at room temperature until all of the substrate had been consumed (TLC). Saturated aqueous $\mathrm{Na}_{2} \mathrm{~S}_{2} \mathrm{O}_{3}$ was added to remove the excess of iodine followed by addition of a potassium fluoride aqueous solution. After stirring at room temperature for $1 \mathrm{~h}$, the resulting white precipitate of tributyltin fluoride was removed by filtration and the filtrate was extracted with ether. The combined organic layers were washed with brine, dried over $\mathrm{MgSO}_{4}$ and concentrated (the crude vinyl iodide could be used in the further step without purification).

(E)-1,3-Dichloro-2-(1-iodo-2-(4-methoxyphenyl)vinyl) benz ene (3a)

Colorless oil (yield $=98 \%$ after filtration through silica gel); $\mathrm{R}_{\mathrm{f}}=0.50\left(\mathrm{Et}_{2} \mathrm{O} /\right.$ Cyclohexane, 5/95 TEA $\left.1 \%, \mathrm{SiO}_{2}\right)$; ${ }^{1} \mathrm{H}$ NMR (300 MHz, $\left.\mathrm{CDCl}_{3}\right): \delta \square 3.65(3 \mathrm{H}, \mathrm{s}), 6.61(2 \mathrm{H}, \mathrm{d}$, $J=8.8 \mathrm{~Hz}), 6.75(2 \mathrm{H}, \mathrm{d}, J=8.8 \mathrm{~Hz}), 7.15(1 \mathrm{H}, \mathrm{t}, J=8.8$ $\mathrm{Hz}), 7.28(2 \mathrm{H}, \mathrm{d}, J=8.5 \mathrm{~Hz}),, 7.39(\mathrm{~s}, 1 \mathrm{H}) .{ }^{13} \mathrm{C}$ NMR $(75$ $\left.\mathrm{MHz}, \mathrm{CDCl}_{3}\right) \delta 55.3\left(\mathrm{CH}_{3}\right), 86.5(\mathrm{C}), 114.0(2 \mathrm{CH}), 128.7$ $(2 \mathrm{CH}), 129,1(\mathrm{C}), 129.6(2 \mathrm{CH}), 130.0(\mathrm{CH}), 134.2(2 \mathrm{C})$, $139.6(\mathrm{C}), 143.9(\mathrm{CH}), 159.5(\mathrm{C}) . \mathrm{IR}\left(\mathrm{cm}^{-1}\right.$, neat): 3077, 2955, 2837, 1602, 1574, 1554, 1509, 1459, 1428, 1355, 1308, 1294, 1260, 1179, 1169, 1151, 1114, 1092, 1034, 951, 935, 891, 878, 816, 780, 739, 679 . 
Methyl 2-((E)-2-(4-((E)-6-hydroxy-1-iodohex-1-enyl)phenyl)-1-iodovinyl)-3,4,5-trimethoxybenzoate (3b)

Following the above general procedure (C), using vinylstannane $20(1.3 \mathrm{~g}, 1.29 \mathrm{mmol})$, DCM $(40 \mathrm{~mL})$ as solvent, $\mathrm{I}_{2}$ solution (2.2 eq., $723 \mathrm{mg}, 2.85 \mathrm{mmol}$ ) in DCM $(40 \mathrm{~mL})$. The reaction was stirred at $-40{ }^{\circ} \mathrm{C}$ for $4 \mathrm{~h}$ in the dark.

Yellow oil (yield $=98 \%$ after filtration through silica gel); $\mathrm{R}_{\mathrm{f}}=0.37$ (AcOEt/Cyclohexane, 20/80 TEA 1\%, $\mathrm{SiO}_{2}$ ); ${ }^{1} \mathrm{H}$ NMR (300 MHz, $\left.\mathrm{CDCl}_{3}\right): \delta \square 1.21(4 \mathrm{H}, \mathrm{m}), 1.76(2 \mathrm{H}, \mathrm{q}, J$ $=7.4 \mathrm{~Hz}), 3.35(2 \mathrm{H}, \mathrm{t}, J=5.7 \mathrm{~Hz}), 3.61(3 \mathrm{H}, \mathrm{s}), 3.67(3 \mathrm{H}$, s), $3.69(3 \mathrm{H}, \mathrm{s}), 3.78(3 \mathrm{H}, \mathrm{s}), 6.21(1 \mathrm{H}, \mathrm{t}, J=7.4 \mathrm{~Hz}), 6.62$ $(2 \mathrm{H}, \mathrm{d}, J=8.6 \mathrm{~Hz}), 6.85(2 \mathrm{H}, \mathrm{d}, J=8.7 \mathrm{~Hz}), 7.14(1 \mathrm{H}, \mathrm{s})$, $7.29(1 \mathrm{H}, \mathrm{s}) .{ }^{13} \mathrm{C} \mathrm{NMR}\left(75 \mathrm{MHz}, \mathrm{CDCl}_{3}\right): \delta 18.9\left(\mathrm{CH}_{2}\right)$, $32.6\left(2 \mathrm{CH}_{2}\right), 46.1\left(\mathrm{CH}_{2}\right), 51.3\left(\mathrm{CH}_{3}\right), 55.6\left(\mathrm{CH}_{3}\right), 59.9$ $\left(\mathrm{CH}_{3}\right), 60.1\left(\mathrm{CH}_{3}\right), 60.2\left(\mathrm{CH}_{2}\right), 91.6(\mathrm{C}), 94.3(\mathrm{C}), 108.7$ $(\mathrm{CH}), 123.9$ (2 CH), $127.4(\mathrm{C}), 128.5(\mathrm{C}), 131.2(2 \mathrm{CH})$, $136.8(\mathrm{CH}), 140.5(\mathrm{CH}), 145.9(\mathrm{C}), 150.1(\mathrm{C}), 153.2(2 \mathrm{C})$, $170.9(\mathrm{C}=\mathrm{O}) . \mathrm{IR}\left(\mathrm{cm}^{-1}\right.$, neat $): 3406,2937,2857,1725$, $1585,1489,1451,1431,1393,1333,1255,1223,1194$, $1171,1116,1052,755$.

Typical Procedure for Negishi coupling between OrthoOrtho' vinyliodide and organozinc compounds (D).

To a solution of vinyl iodide $2, \mathrm{PdCl}_{2}\left(\mathrm{PPh}_{3}\right)_{2}(5 \mathrm{~mol} \%)$ in THF was added at room temperature $\mathrm{ArZnCl}$ ( 2 equiv.) prepared by transmetallation from the corresponding Grignard reagent (2 equiv.) and anhydrous $\mathrm{ZnCl}_{2}$ (2.1 equiv.). The reaction was stirred at room temperature and monitored by TLC until complete consumption of starting materials $\left(2\right.$ to $4 \mathrm{~h}$ ). The reaction was hydrolyzed at $0^{\circ} \mathrm{C}$ with aqueous $\mathrm{HCl}(1 \mathrm{~N})$, extracted with $\mathrm{Et}_{2} \mathrm{O}$, the organic extract was dried over $\mathrm{MgSO}_{4}$ and the solvent was removed in vacuo.

(Z)-4,4'-(1-(2,6-Dichlorophenyl)ethene-1,2-

diyl)bis(methoxybenzene) (4a)

colorless oil $($ yield $=73 \%) ; \mathrm{R}_{\mathrm{f}}=0.29\left(\mathrm{Et}_{2} \mathrm{O} /\right.$ Cyclohexane, 5/95, $\left.\mathrm{SiO}_{2}\right) ;{ }^{1} \mathrm{H}$ NMR $\left(300 \mathrm{MHz}, \mathrm{CDCl}_{3}\right): \delta \square 3.66(3 \mathrm{H}, \mathrm{s})$, $3.73(3 \mathrm{H}, \mathrm{s}), 6.63(2 \mathrm{H}, \mathrm{d}, J=8.9 \mathrm{~Hz}), 6.79(2 \mathrm{H}, \mathrm{d}, J=8.9$ $\mathrm{Hz}), 6.84(2 \mathrm{H}, \mathrm{d}, J=8.9 \mathrm{~Hz}), 6.99(1 \mathrm{H}, \mathrm{s}), 7.22-7.11(3 \mathrm{H}$, $\mathrm{m}), 7.31(2 \mathrm{H}, \mathrm{d}, J=7.7 \mathrm{~Hz}) .{ }^{13} \mathrm{C} \mathrm{NMR}\left(75 \mathrm{MHz}, \mathrm{CDCl}_{3}\right): \delta$ $55.3\left(\mathrm{CH}_{3}\right), 55.4\left(\mathrm{CH}_{3}\right), 113.8(2 \mathrm{CH}), 114.1(2 \mathrm{CH}), 127.2$ $(2 \mathrm{CH}), 128.6(2 \mathrm{CH}), 128.6(\mathrm{CH}), 129.4(\mathrm{CH}), 129.7$ (2CH), 129.8 (C), 132.8 (C), 133.9 (C), 135.7 (2C), 138.4 (C), $158.8(\mathrm{C}), 159.2$ (C). IR (cm ${ }^{-1}$, neat): 3001, 2955, 2932, 2900, 1606, 1573, 1555, 1511, 1462, 1441, 1427, 1366, 1302, 1288, 1256, 1241, 1175, 1153, 1113, 1090, 1031, 908, 850, 827, 783, 767, 754, 681. Anal. Calcd for $\mathrm{C}_{22} \mathrm{H}_{18} \mathrm{Cl}_{2} \mathrm{O}_{2}$ (385.28): $\mathrm{C}$ 68.58, $\mathrm{H}$ 4.71, found $\mathrm{C} 68.62, \mathrm{H}$ 4.54 .

Methyl 2-( (Z)-2-( 4-((E)-6-hydroxy-1-( 4-methoxyphenyl) hex-1-enyl)phenyl)-1-(4-methoxyphenyl)vinyl)-3,4,5-trimethoxybenzoate (4b)

Following the above general procedure (D), using bromo (4-methoxyphenyl)zinc (6 eq.) in THF, $\mathrm{PdCl}_{2}\left(\mathrm{PPh}_{3}\right)_{2}(5 \%)$ and 31 (1 eq.). The reaction was stirred at room tempreature for $2 \mathrm{~h}$ in the dark.

Yellow oil (yield $=61 \%) ; \mathrm{R}_{\mathrm{f}}=0.10($ AcOEt/Cyclohexane, $\left.30 / 70, \mathrm{SiO}_{2}\right) ;{ }^{1} \mathrm{H}$ NMR (400 MHz, $\left.\mathrm{CDCl}_{3}\right): \delta \square 1.44(4 \mathrm{H}$, $\mathrm{m}), 1.62(2 \mathrm{H}, \mathrm{m}), 2.01(1 \mathrm{H}, \mathrm{brs}, \mathrm{OH}), 3.71(2 \mathrm{H}, \mathrm{m}), 3.76$ $(6 \mathrm{H}, \mathrm{s}), 3.81(3 \mathrm{H}, \mathrm{s}), 3.91(3 \mathrm{H}, \mathrm{s}), 3.94(3 \mathrm{H}, \mathrm{s}), 3.97(3 \mathrm{H}$, s), $6.08(1 \mathrm{H}, \mathrm{t}, J=7.8 \mathrm{~Hz}), 6.87-6.91(12 \mathrm{H}, \mathrm{m}), 7.12(1 \mathrm{H}$, s), $7.23(1 \mathrm{H}, \mathrm{s}) .{ }^{13} \mathrm{C}$ NMR $\left(100 \mathrm{MHz}, \mathrm{CDCl}_{3}\right): \delta 25.9$ $\left(\mathrm{CH}_{2}\right), 29.1\left(\mathrm{CH}_{2}\right), 31.9\left(\mathrm{CH}_{2}\right), 51.1\left(\mathrm{CH}_{3}\right), 59.9\left(\mathrm{CH}_{3}\right)$, $60.5\left(\mathrm{CH}_{3}\right), 60.7\left(\mathrm{CH}_{3}\right), 62.3\left(\mathrm{CH}_{2}\right), 109.4(\mathrm{CH}), 113.3$ $(2 \mathrm{CH}), 113.5(2 \mathrm{CH}), 127.8(2 \mathrm{CH}), 127.5(2 \mathrm{CH}), 128.1$ $(2 \mathrm{C}), 129.4(\mathrm{C}), 135.3(2 \mathrm{CH}), 135.5(2 \mathrm{CH}), 135.8(\mathrm{C})$, $136.4(2 \mathrm{C}), 136.7(\mathrm{C}), 138.5(\mathrm{C}), 139.0(\mathrm{CH}), 140.9(\mathrm{CH})$, $152.6(\mathrm{C}), 158.6$ (2C), $158.8(2 \mathrm{C}), 167.3(\mathrm{C}=\mathrm{O})$. IR $\left(\mathrm{cm}^{-1}\right.$, neat): 2940, 2250, 1726, 1510, 1335, 1115, 910, 730; Anal. Calcd for $\mathrm{C}_{39} \mathrm{H}_{42} \mathrm{O}_{8}$ (638.75): C 73.33, H 6.63, found $\mathrm{C}$ 73.42, H 6.32 .

\section{General procedure for the hydrosilylation of alkynes (E).}

In a sealed tube, $\mathrm{PtO}_{2}(11.35 \mathrm{mg}, 0.05 \mathrm{mmol})$ and alkyne (1 mmol) were placed under nitrogen atmosphere. Triethylsilane $(0.24 \mathrm{~mL}, 1.5 \mathrm{mmol})$ was introduced via syringe and the mixture was stirred at $60^{\circ} \mathrm{C}$ in an oil bath for $1 \mathrm{~h}$. in the absence of solvent. The residue was then purified by column chromatography over silica gel to yield the title adducts either as a single $\alpha$-isomer $\mathbf{5}$ or as a mixture of inseparables $\mathbf{5} \boldsymbol{\alpha}$ and $\mathbf{5} \boldsymbol{\beta}$-isomers.

(E)-(1-(2,6-Dimethoxyphenyl)-2-phenylvinyl)triethylsilane $(\boldsymbol{\alpha}-\mathbf{5 a})$. colorless oil (yield $=90 \%$ after filtration through silica gel); $\mathrm{R}_{\mathrm{f}}=0.70$ (AcOEt/Cyclohexane, 20/80, $\mathrm{SiO}_{2}$ ); Major $\boldsymbol{\alpha}$ isomer : ${ }^{1} \mathrm{H} \mathrm{NMR}\left(300 \mathrm{MHz}, \mathrm{CDCl}_{3}\right) \delta 0.61(6 \mathrm{H}$, q, $J=6.1 \mathrm{~Hz}), 0.93(9 \mathrm{H}, \mathrm{t}, J=6.2 \mathrm{~Hz}), 3.63(6 \mathrm{H}, \mathrm{s}), 6.52$ $(2 \mathrm{H}, \mathrm{d}, J=8.3 \mathrm{~Hz}), 6.85(1 \mathrm{H}, \mathrm{s}), 7.12-6.99(5 \mathrm{H}, \mathrm{m}), 7.16$ $(1 \mathrm{H}, \mathrm{t}, J=8.3 \mathrm{~Hz}) \cdot{ }^{13} \mathrm{C} \mathrm{NMR}\left(75 \mathrm{MHz}, \mathrm{CDCl}_{3}\right) \delta 3.7$ $\left(3 \mathrm{CH}_{2}\right), 7.4\left(3 \mathrm{CH}_{3}\right), 55.6\left(2 \mathrm{CH}_{3}\right), 104.0(2 \mathrm{CH}), 120.2(\mathrm{C})$, $126.8(\mathrm{CH}), 127.1(\mathrm{CH}), 127.9(2 \mathrm{CH}), 128.5(2 \mathrm{CH}), 136.2$ (C), $138.9(\mathrm{C}), 139.6(\mathrm{CH}), 156.5$ (2C). IR ( $\mathrm{cm}^{-1}$, neat): 2950, 2875, 1583, 1468, 1431, 1246, 1110, 1006, 907, 781, 762, 649. Anal. Calcd for $\mathrm{C}_{22} \mathrm{H}_{30} \mathrm{O}_{2} \mathrm{Si}$ (354.56): $\mathrm{C} 74.53, \mathrm{H}$ 8.53, found C 74.41, H 8.44.

\section{(E)-(1-(2,6-Dimethoxyphenyl)-2-(4-methoxyphenyl)vinyl)} triethylsilane $(\mathbf{\alpha - 5} \mathbf{b})$

Colorless oil (yield $=97 \%$ after filtration through silica gel); $\mathrm{R}_{\mathrm{f}}=0.65\left(\mathrm{AcOEt} / \mathrm{Cyclohexane}, 20 / 80, \mathrm{SiO}_{2}\right)$; Major $\boldsymbol{\alpha}$ isomer : ${ }^{1} \mathrm{H} \mathrm{NMR}\left(300 \mathrm{MHz}, \mathrm{CDCl}_{3}\right) \delta 0.58(6 \mathrm{H}, \mathrm{q}, J=$ $7.8 \mathrm{~Hz}), 0.93(9 \mathrm{H}, \mathrm{t}, J=7.8 \mathrm{~Hz}), 3.65(6 \mathrm{H}, \mathrm{s}), 3.72(3 \mathrm{H}, \mathrm{s})$, $6.54(2 \mathrm{H}, \mathrm{d}, J=8.3 \mathrm{~Hz}), 6.64(2 \mathrm{H}, \mathrm{d}, J=8.8 \mathrm{~Hz}), 6.78(1 \mathrm{H}$, s), $6.99(2 \mathrm{H}, \mathrm{d}, J=8.8 \mathrm{~Hz}), 7.16(1 \mathrm{H}, \mathrm{t}, J=8.3 \mathrm{~Hz}) .{ }^{13} \mathrm{C}$ NMR $\left(75 \mathrm{MHz}, \mathrm{CDCl}_{3}\right) \delta 3.7\left(3 \mathrm{CH}_{2}\right), 7.4\left(3 \mathrm{CH}_{3}\right), 55.2$ $\left(\mathrm{CH}_{3}\right), 55.6\left(2 \mathrm{CH}_{3}\right), 104.1(2 \mathrm{CH}), 113.4(2 \mathrm{CH}), 120.4(\mathrm{C})$, $127.0(\mathrm{CH}), 129.8(2 \mathrm{CH}), 131.9(\mathrm{C}), 133.5(\mathrm{C}), 138.8$ (CH), 156.6 (2C), 158.5 (C). IR ( $\mathrm{cm}^{-1}$, neat): 2950, 1508, 1468, 1432, 1246, 1176, 1111, 1036, 1007, 905, 762, 727,696, 650. Anal. Calcd for $\mathrm{C}_{23} \mathrm{H}_{32} \mathrm{O}_{3} \mathrm{Si}$ (384.58): C 71.83, H 8.39, found C 71.49, H 8.32. 


\section{(E)-(1-(2,6-Dichlorophenyl)-2-phenylvinyl)triethylsilane} $(\alpha-5 c)$

Colorless oil (yield $=98 \%$ after filtration through silica gel); $\mathrm{R}_{\mathrm{f}}=0.40$ (Cyclohexane, $\left.\mathrm{SiO}_{2}\right) ;{ }^{1} \mathrm{H}$ NMR $(300 \mathrm{MHz}$, $\left.\mathrm{CDCl}_{3}\right) \delta 0.72(6 \mathrm{H}, \mathrm{q}, J=6.1 \mathrm{~Hz}), 0.96(9 \mathrm{H}, \mathrm{t}, J=6.1 \mathrm{~Hz})$, 7.18-6.88 (6H, m), 7.30-7.26 (3H, m). ${ }^{13} \mathrm{C}$ NMR (75 MHz, $\left.\mathrm{CDCl}_{3}\right) \delta 4.4\left(3 \mathrm{CH}_{2}\right), 7.4\left(3 \mathrm{CH}_{3}\right), 127.6(\mathrm{CH}), 127.7(\mathrm{CH})$, $128.2(\mathrm{CH}), 128.3(2 \mathrm{CH}), 133.4(2 \mathrm{C}), 137.7$ (C), 140.7 (C), $141.5(2 \mathrm{CH}), 148.2$ (C). IR ( $\mathrm{cm}^{-1}$, neat): 2954, 2875, 1426, 1009, 965, 937, 777, 765, 749, 730, 717, 688. Anal. Calcd for $\mathrm{C}_{20} \mathrm{H}_{24} \mathrm{Cl}_{2} \mathrm{Si}$ (363.40): C 66.10, H 6.66, found C 66.05, H 6.45 .

\section{Acknowledgments}

We thank the CNRS for support this research.

\section{References}

1 (a) Peyrere, M.; Quintard, J.-P.; Rahm, A. Tin in Organic Synthesis; Butterworth: London, 1987. (b) Farina, V.; Krishnamurthy, V.; Scott, W. J. Org. React. 1997, 50, 1 and references therein. (c) Shirakawa, E.; Nakao, Y.; Tsuchimoto, T.; Hiyama, T. Chem. Commun. 2002, 1962.

2 (a) Tolstikov, G. A.; Miftakhov, M. S.; Danilova, N. A.; Vel'Der Y. L. Synthesis 1986, 496. (b) Nativi, C.; Taddei, M. J. Org. Chem. 1988, 53, 820. (c) Miyake, H.; Yamamura, K. Chem. Lett. 1989, 981. (d) Zhang, H. X.; Guibé, F.; Balavoine G. J. Org. Chem. 1990, 55, 1857. (e) Stille, J. K. Angew. Chem., Ed. Engl. 1986, 25, 508. (f) Chu, K. H.; Wang, K. K. J. Org. Chem. 1986, 51, 767. (g) Lautens, M.; Huboux, A. H. Tetrahedron Lett. 1990, 31, 3105. (h) Marshall, J. A.; Bourbeau, M. P.Org. Lett. 2002, 4, 3931.

3 (a) Miyake, H.; Yamamura, K. Chem. Lett. 1989, 981. (b) Zhang, H. X.; Guibé, F.; Balavoine, G. J. Org. Chem. 1990, 55, 1857. (c) Smith, N. D.; Mancuso, J.; Lautens M. Chem. Rev. 2000, 100, 3257. (d) Trost, B. M.; Ball, Z. T. Synthesis 2005, 853.

4 Betzer, J. F.; LeMenez, P.; Prunet, J.; Brion, J.-D.; Ardisson, J.; Pancrazi, A. Synlett 2002, 1.

5 (a) Greeves, N.; Torode, J. S Synlett 1994, 537. (b) Semmelhack, M. F.; Hooley, R. J. Tetrahedron Lett. 2003, 44, 5737. (c) Marshall, J. A.; Bourbeau, M. P.Tetrahedron Lett. 2003, 44, 1087. (d) Oikawa, H.; Yoneta, Y.; Ueno, T.; Oikawa, M.; Wakayama, W.; Ichihara, A. Tetrahedron Lett. 1997, 38, 7897.

6 (a) Magriotis, P. A.; Brown, J. T.; Scott, M. E. Tetrahedron Lett. 1991, 32, 5047. (b) Huang, X.; Ma, Y. Synthesis 1997, 417. (c) Buissonneaud, D.; Cintrat, J.-C. Tetrahedron Lett. 2006, 47, 3139. (d) Casson, S.; Kocienski, P.Synthesis 1993, 1133. (e) Boden, C. D. J.; Pattenden, G.; Ye T. J. Chem. Soc., Perkin Trans. 1 1996, 2417. (f) Paley, R. S.; Weers, H. L.; Fernandez, P. Tetrahedron Lett. 1995, 36, 3605.

7 (a) Alami, M.; Ferri, F. Synlett 1996, 755. (b) Hamze, A.; Provot, O.; Brion, J.-D.; Alami, M. J. Org. Chem. 2007, 72, 3868.

8 (a) Ferri, F.; Alami, M. Tetrahedron Lett. 1996, 37, 7971. (b) Bujard, M.; Ferri, F.; Alami M. Tetrahedron Lett. 1998, 39, 4243.

9 (a) Liron, F.; Le Garrec, P.; Alami, M.Synlett. 1999, 246. (b) Alami, M.; Liron, F.; Gervais, M.; Peyrat, J.-F.; Brion, J.-D. Angew. Chem., Int. Ed. 2002, 41, 1578. (c) Liron, F.; Gervais, M.; Peyrat, J.-F.; Alami, M.; Brion J.-D. Tetrahedron Lett. 2003, 44, 2789.

10 Hamze, A.; Veau, D.; Provot, O., Brion, J.-D.; Alami, M. J. Org. Chem. 2009, 74, 1337.

11 Hamze, A.; Provot, O.; Brion, J.-D.; Alami M. Org. Lett. 2005, 7, 5625 .
12 (a) Sonogashira, K.; Tohda, Y.; Hagihara, N. Tetrahedron Lett. 1975, 4467. (b) Alami, M.; Ferri, F.; Linstrumelle G. Tetrahedron Lett. 1993, 34, 6403.

13 For diaryl derivatives, $\alpha$ isomers have been fully identified by NOESY experiments. e.g. for $\mathbf{2 b}$

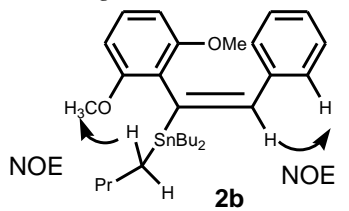

14 a) Liron, F.; Gervais, M.; Peyrat, J.-F.; Alami, M.; Brion, J.-D. Tetrahedron Lett., 2003, 44, 2789. b) Bujard, M.; Ferri, F.; Alami, M. Tetrahedron Lett., 1998, 39, 4243.

15 See for examples: (a) Tanpure, R. P.; Harkrider, A. R.; Strecker, T. E.; Hamel, E.; Trawick, M. L.; Pinney, K. G. Bioorg. Med. Chem. 2009, 17, 6993. (b) Harper, M. J. K.; Walpole, A. L. Nature 1966, 212, 87. (c) Rasolofonjatovo, E.; Provot, O.; Hamze, A.; Bignon, J.; Brion, J.-D.; Alami, M. Eur. J. Med. Chem. 2010, in press.

16 Hayashi, K.; Iyoda, J.; Shiihara I. J. Organomet. Chem. 1967, 10, 81 . 